\title{
Competitive markdown timing for perishable and substitutable products is $^{2}$
}

\author{
Alper Şen \\ Department of Industrial Engineering, Bilkent University, Bilkent, Ankara 06800, Turkey
}

\section{A R T I C L E I N F O}

\section{Article history:}

Received 12 September 2014

Accepted 29 October 2015

Available online 10 November 2015

\section{Keywords:}

Pricing competition

Revenue management

Timing game

\begin{abstract}
A B S T R A C T
We model as a duopoly two firms selling their fixed stocks of two substitutable items over a selling season. Each firm starts with an initial price, and has the option to decrease the price once. The problem for each firm is to determine when to mark its price down in to maximize its revenue. We show that the existence and characterization of a pure-strategy equilibrium depend on the magnitude of the increase in the revenue rate of a firm when its competitor runs out of stock. When the increase is smaller than the change in the revenue rate of the price leader when both firms are in stock for all of the three possible scenarios, neither firm has the incentive to force its rival to run out of stock and if a firm marks its price down after the season starts, its inventory runs out precisely at the end of the season. When the increase is larger than the change of the price leader's revenue rate in one particular scenario, waiting until its rival runs out of inventory may be an equilibrium strategy for the larger firm even though this may lead to leftover inventory for itself. In other cases, there may be no pure-strategy equilibrium in the game. In certain regions of the parameter space, a firm's revenue may be decreasing in its starting inventory which shows that a firm may be better off if it can credibly salvage a portion of its inventory prior to the game. While most of our analysis is for open-loop strategies, in the final part of the paper, we show that the open-loop equilibrium survives as an equilibrium when we consider closed-loop strategies for an important subset of the parameter space.
\end{abstract}

(c) 2015 Elsevier Ltd. All rights reserved.

\section{Introduction}

Many companies in various industries face the problem of selling a fixed amount of inventory over a finite horizon. Examples include retailers selling perishable goods such as apparel, electronics and toys, airlines selling a fixed number of airplane seats, and hotels selling a fixed number of rooms. A frequent reason for fixed inventories is the lack of replenishment opportunities due to relatively long replenishment lead times as compared to the length of the selling season. For example, according to a recent survey, average lead time in the apparel and footwear industries is 11 months [3], while the fashion seasons themselves are as short as 2-3 months [28]. This situation leads to retailers ordering most or all of their merchandise prior to the season. For these retailers, pricing is the only control to match supply and demand once they place their orders. According to one estimate by a consulting firm, a typical retailer sells between 40 and 45 percent of its merchandise at a discounted price [35]. A vivid example is J.C. Penney, a major US department store, which generates 73 percent of its revenue from products sold at a discount of 50 percent or more, and only 0.2 percent from goods bought at full price [20]. Long lead times and hard-to-predict demand also cause toy retailers using excessive markdowns to match supply and demand [38]. Markdowns are dominant in the auto industry, where manufacturers introduce a new vintage of a vehicle every year. According to a study by Copeland et al. [5], the price of a new vehicle declines by 9.2 percent over the model year, and half of these declines are driven by promotions to clear the inventory that dealers and factories build up of that model year's vehicles. For example, Ford had 103 days of supply or 27,100 units of 2006 Expeditions at the beginning of July, 2006. The 2007 model was to be launched in September, so the company initiated a promotion in that summer and offered a discount between $\$ 5,000$ and $\$ 6,000$ per vehicle to clear its inventory [1].

Markdowns may have a dramatic effect on a retailer's profitability. Many retailers blame excessive markdowns to their recent financial troubles [34]. At the same time, managing markdowns can be challenging since marking the price down too early or too deeply will lead to

\footnotetext{
${ }^{2}$ This manuscript was processed by Associate Editor Y. Wang.

E-mail address: alpersen@bilkent.edu.tr
} 
lost revenue, while delaying markdowns or keeping them shallow will lead to liquidating inventory at even lower prices at the end of the season. Due to such challenges, many retailers have begun using markdown optimization software to determine the timing, depth and frequency of their clearance or markdown events. Software vendors that offer markdown optimization solutions include DemandTec, Oracle, Predictix, Revionics and SAS [31].

As in many other areas of business, firms are usually very sensitive to the pricing activities of their competitors. For example, in 2010, Best Buy, the largest electronics retailer in the US, started its holiday sales 10 days earlier than previous years considering competition in addition to its inventory build-up [36]. In the auto industry, competition (and inventory as discussed above) also shapes year-end clearance offers [2].

In this paper, we study a markdown competition game between two firms whose two products are substitutes for each other. Each firm is endowed with a fixed amount of inventory that it needs to sell over a common selling season. We assume that the firms are symmetric except for their starting inventory levels and assume deterministic demands. One firm's demand rate at a given time depends on its own price as well as its competitor's price and stock availability. We assume no particular function to define this dependency, except that the demand is decreasing in own price and increasing in its competitor's and that unilateral price drops are revenue increasing. Each firm starts the selling season with a common high price and has a single chance to switch to a lower one (which is also common) during the season. With this simplification, the game we study is a simple finite horizon timing game, in which each firm's strategy is the time of its markdown. We explore two types of equilibria in this setting. First, we assume that the firms pre-commit themselves to the markdown times at the beginning of the season and use a static game to explore the strategic interactions between the firms. In this case, we identify the open-loop equilibrium of the game. While the assumptions regarding exogenous markdown prices and pre-commitment to markdown times may be too restrictive in many practical settings, they may be justified for a limited number of firms that practice what is known as pre-announced or automatic markdowns. In this strategy, the seller announces the future prices (which are usually fixed percentages of the original price) along with the times that these prices will take effect (provided that there is still inventory) in advance. Examples include Land's End Overstocks for fashion apparel, Tesco's Fresh \& Easy for groceries and Theater Development Fund's TKTS for theater tickets.

In the final part of the paper, for an important subset of the parameter space, we assume that firms can observe each other's actions throughout the selling season and dynamically decide when to mark the price down. In this case, we use subgame-perfect equilibrium as a solution concept and identify the closed-loop equilibrium of the game and show that this coincides with the open-loop equilibrium. That is, for this region of parameter space, the firms do not have any incentive to preempt or wait for each other in marking the prices down during the season; their decisions at the beginning of the season do not change.

We also assume away the stochastic nature of demand in these settings. However, note that the markdown decisions are made after the actual season starts, i.e., when, in practice, a considerable portion of the uncertainty is resolved and accuracy of demand forecast is reasonably high (see, for example, [8]).

We find that the existence of a pure-strategy equilibrium and its characterization critically depend on the maximum demand rate that a firm faces when its competitor runs out of stock (i.e., monopoly demand rate when the price is low) relative to three thresholds. These thresholds are functions of the demand rates and prices and measure the effectiveness of price changes when the competitor is in stock relative to when the competitor is out of stock. If the monopoly demand rate is smaller than all thresholds, there is a pure-strategy equilibrium in the game, and each firm's equilibrium markdown time can be characterized as a function of starting inventory levels and length of the selling season. The equilibrium is one of seven possible equilibria, depending on where the starting inventory levels and the selling season fall in the parameter space. In all equilibria, the larger firm (the firm with the larger starting inventory) always marks its price down earlier than the smaller firm. We show that each firm either (i) marks down the price at the beginning of the season (ii) never marks the price down (iii) marks the price down in the middle of the season at such a time that its inventory runs out precisely at the end of the season. In other words, it is not an equilibrium strategy to change the price after the season starts and still have some leftover inventory at the end of the season, or run out of stock before the season ends.

When the monopoly demand rate is larger than the last threshold but smaller than the first two, a pure-strategy Nash equilibrium still exists. The equilibrium in this case is one of six possible equilibria. Different from the previous case, in one of the equilibria, the larger firm may wait for the smaller firm to exhaust its stock, and switch immediately after, even though this may lead to leftover inventory at the end of the season. We also derive a set of sufficient conditions for the uniqueness of the equilibria in these cases. Finally, we show by examples that if the monopoly demand rate is larger than one of the first two thresholds, a pure-strategy Nash equilibrium may fail to exist.

We show that the three thresholds mentioned above are never exceeded and the uniqueness conditions are easily satisfied if the demand rates originate from two important demand models: linear demand model and attraction demand model.

Under a single-firm setting, the revenue is monotone increasing in the starting inventory level and the length of the selling season. Markdown time, on the other hand, is monotone decreasing in the starting inventory level and increasing in the length of the selling season. One would expect these results to carry to the competitive case. Another intuitive conjecture for the competitive case is that a firm's revenue and markdown time are monotone decreasing in its competitor's starting inventory level. Interestingly, comparative statics results of the competitive game lead to exceptions to these properties. First, firms' payoffs are not monotone increasing in their own starting inventories, particularly when the firms have intermediate levels of starting inventory and when their demands are inelastic to an industry-wide markdown. In this case and under the assumptions of our model, both firms' revenues may be decreasing in their starting inventory levels and they are better off if they can credibly salvage some of their inventory prior to the game. Alternatively, firms will not prefer to have more inventory even if it was free. When one firm is substantially small and the larger firm has an incentive to wait until the smaller firm exhausts its stock, the smaller firm's revenue jumps down as its starting inventory goes up, again breaking the monotonicity of the smaller firm's payoff in its own inventory. In this equilibrium, the larger firm's markdown time is no longer monotone decreasing in the smaller firm's inventory, either.

Under a reasonable assumption that the starting inventory levels of both firms are bounded from below (above) by what they can sell when both firms are charging a high (low) price, we show that the length of the period during which firms charge different prices increases linearly with inventory imbalance (measured as the difference between starting inventory levels) and decreases reciprocally with product substitution (measured as the difference between the demand rates of firms when they charge different prices). 
The rest of the paper is organized as follows: In Section 2, we review the related literature on competitive pricing and revenue management. In Section 3, we explain our model. In Section 4, we characterize the equilibrium for the competitive markdown timing game and in Section 5, we derive comparative statics results. We provide the analysis for closed-loop equilibrium in Section 6 . We conclude the paper in Section 7 and present some avenues for future research.

\section{Literature survey}

This paper is part of the revenue management literature which studies how a firm should set and update pricing and product availability decisions over a finite horizon to maximize the revenue from a perishable asset. Revenue management has grown from its origins in airlines and other services to become an important practice in various industries including retailing and manufacturing. For a thorough review of research and applications in this important area, the reader should consult the book by Talluri and van Ryzin [33].

An important branch of revenue management is price-based revenue management, which uses price as the key control. A seminal work in this area is by Gallego and van Ryzin [11], who study the pricing decisions of a firm selling a fixed stock of items over a finite horizon under Poisson demand. They show that the optimal profit of the deterministic problem provides an upper bound for the optimal expected profit and fixed-price heuristics for this problem are asymptotically optimal. Gallego and van Ryzin [12] study the multi-product case; they suggest two asymptotically optimal heuristics and apply them to network revenue management problems. Feng and Gallego [7] have the same setup as Gallego and van Ryzin [11]; however in the former model, prices are given, and the problem is deciding the optimal timing of a single price change from a given initial price to a given lower or higher second price. Our paper can be considered as a competitive version of their markdown model (price change is from high to low) for deterministic demands.

In the economics literature, clearance sales or markdowns have also received some attention. Lazear [17] develops a model of retailing, in which a firm sets the price of a product over a finite number of periods to maximize profit. It is assumed that the consumers are homogeneous (they have the same valuation of the item) and that they shop at once when the price is declared. Pashigian [24] extends Lazear's model to allow for industry equilibrium and shows that fashion and product variety are the leading reasons for the increased use of markdowns in fashion retailing.

There is an extensive economics literature on pricing in oligopolistic markets [39]. A relevant model in this literature is the BertrandEdgeworth competition, where firms offer homogeneous products and may have capacity restrictions such that the total demand cannot be supplied. In this case, the existence of a pure-strategy equilibrium is not guaranteed for the static pricing game. This problem is less severe, but does not go away when the products are differentiated (Bertrand-Edgeworth-Chamberlin competition). In our model also, under certain conditions pure-strategy Nash equilibria may not exist. However, these cases are true exceptions in our model.

Competition is prevalent in many industries where revenue management is practiced, but competitive models of revenue management have only recently appeared in the literature. In price-based revenue management, Perakis and Sood [25] are one of the first to study pricing of fixed inventories in a competitive environment. In their multi-period, deterministic model with non-homogeneous products, sellers pre-commit themselves to prices at the beginning of the horizon (i.e., a static game). The authors show that under certain conditions, there exists a unique equilibrium of the game. A numerical study shows that certain monotonicity results, such as that higher inventory for a firm leads to lower prices for both firms, but higher revenues for that firm and lower revenues for its competitor, hold as expected for their game. An important contribution of our paper is the analytical derivation of monotonicity results and showing that under some conditions, some of the expected monotonicity properties may not hold. Perakis and Sood [26] relax the assumption of deterministic demands, by allowing the demand parameters to take a value in an uncertainty set and use a robust optimization approach to study the problem. Tsai and Hung [37] develop an integrated real options approach for revenue management and dynamic pricing in Internet retailing under competition.

Xu and Hopp [41] study oligopolistic competition in which firms compete on initial inventory levels as well as on prices which are dynamically adjusted during the horizon. They assume that customers arrive following a geometric Brownian motion and choose from the set of lowest priced and in-stock firms based on a logit choice model. Lin and Sibdari [19] study dynamic pricing competition among firms that sell substitutable products. In their periodic model, customers arrive according to a Bernoulli process and their choice is governed by the multinomial logit (MNL) model. The authors provide an example that shows that a firm's price may not be monotone in remaining time in the selling season. The formulation in Gallego and Hu [10] is a stochastic game in continuous time where demand is modeled as a non-homogeneous Poisson process with rates dependent on time and prices posted by all firms through a more general function. For the deterministic version of the game, they derive the conditions for the existence and uniqueness of an open-loop equilibrium and show that this equilibrium is also a feedback equilibrium of the game. An interesting result in this case is that in equilibrium, all firms may not utilize the whole sales season. For the stochastic case, they show that equilibrium of the deterministic game can be used to construct heuristics that are asymptotically equilibrium.

Levin et al. [18] consider the impact of strategic consumer behavior and seller competition in dynamic pricing of fixed inventories. They show that strategic behavior may have substantial impact on firms' revenues. In a recent paper, Martinez-de Albeniz and Talluri [21] study a dynamic pricing competition game with fixed inventories and homogeneous products. In this game, at most one customer arrives in each period, each customer has the same valuation and chooses the lowest-priced retailer. The authors show that a subgame-perfect equilibrium exists and the seller with the lower equilibrium reservation value sells a unit at a price equal to the competitor's equilibrium reservation value. The model is extended to the case of uncertain and time-varying customer valuations. The existence of a unique subgame-perfect equilibrium holds also for the case of differentiated products, but the authors state that obtaining analytic solutions is intractable.

The paper by Whang [40] is the closest to ours as it is the only one that explicitly focuses on timing decisions under competition. Two firms start a selling season with fixed inventories of two substitutable items. Demands are deterministic and follow a specific demand trajectory that peaks at introduction and declines exponentially over time. Each player's strategy in the game is the timing of its single markdown. Firms are symmetric (with common pre-fixed initial and markdown prices and corresponding demand trajectories), except for the initial inventory, which is private information. A strong assumption is that a stock-out in one firm does not affect the demand at the other firm. A particular strategy set is assumed which is represented by two time thresholds. This strategy for the given thresholds can be 
described as follows: "Do not mark the price down until the first threshold; mark down immediately after the competitor between the first and second thresholds; and mark the price down at the second threshold if the competitor has not marked the price down until that time". This model does not lead to a closed-form solution and therefore no managerial insights were available from the analysis. The author states: "We could not obtain any crisp results from the present simple model, I would rather hope to see a model that is even simpler and yet insightful". Our paper follows the lead of Whang [40] and provides a full characterization of the equilibrium of the markdown competition game under the assumptions of complete information and pre-commitment to markdown times. In contrast to Whang [40], however, we model customer behavior when one firm runs out of stock. This is a key construct in our model and plays an important role in the existence and characterization of the equilibrium. In some of our possible equilibria, the larger firm enjoys monopoly power after its competitor runs out of stock. Ghemawat and McGahan [13] provide evidence of this behavior from the turbine generator industry.

There is also a growing literature on competitive models in quantity-based revenue management. See Grauberger and Kimms [16] and references therein. Finally, the game in our study is a timing game. Timing games are used extensively in the economics literature to understand issues such as adoption of new technology [27] and exit from declining industries [14].

\section{The model}

Our model is an initial attempt to understand the effects of strategic interactions on pricing decisions of firms in the presence of inventory considerations. Therefore, we make a few simplifying assumptions about the structure of the problem, some of which can be partially justified based on the previous results of Gallego and van Ryzin [11]. First, although the magnitude of these markdowns might be equally important, we focus only on their timing and as in Feng and Gallego [7] and Whang [40], we assume that the initial price and the markdown price are exogenous and known in advance. We allow each company to make at most one price change. This restriction may be justified when the costs associated with price changes are considered. Moreover, Gallego and van Ryzin [11] show that a single price change may be as effective as more flexible pricing, especially when the sales volume is high and price changes are costly (See also [29]).

Because of the complex structure of the stochastic solution for even the single-firm case [7], and given that our main purpose is to study the effects of competition, we use deterministic demand rates in this work. This may not be very restrictive when we consider firms making markdown decisions a few weeks after the actual selling season starts when they can better predict demand.

We consider two firms, $A$ and $B$, selling a perishable product in a competitive market. There is a horizon of length $t$ over which these products can be sold (while we sometimes use the term selling season for the period $[0, t]$ in the remainder of the paper, time 0 may correspond to a time later than the start of the fashion season). Both firms start with the same price $p_{1}$ and each firm has a single chance to decrease the price to $p_{2}$ at some time within the horizon. Firms face deterministic demand rates based on the prices charged and the availability of stock at each firm. These demand rates (in the order of firm $A$, and firm $B$ ) are given in Table 1 . As seen in Table 1 , we assume that the firms are symmetric in terms of market power, and thus have symmetric demand rates. Both firms face a demand rate of $\lambda_{1}$ if they are both charging the high price $p_{1}$, and a demand rate of $\lambda_{2}$ if they are both charging the low price $p_{2}$. Subscripts $L$ and $F$ are used to indicate the price leader and the price follower, respectively. The firm which switches to the low price first is called the leader in the price switch game. The price leader faces a demand rate of $\lambda_{L}$ until its competitor also switches its price or runs out of stock. During this time, the other firm, the follower, faces a demand rate of $\lambda_{F}$. If one firm runs out of stock, the other firm receives a demand rate of $\lambda_{M 1}$ at price $p_{1}$ and $\lambda_{M 2}$ at price $p_{2}$.

The demand rates provided in Table 1 are general in the sense they can originate from any price-response function. However, we require the following obvious assumptions.

Assumption 1. The demand rates satisfy

(a) $\lambda_{F} \leq \lambda_{1} \leq \lambda_{M 1}$,

(b) $\lambda_{2} \leq \lambda_{L} \leq \lambda_{M 2}$,

(c) $\lambda_{1} \leq \lambda_{2}$.

Assumption 1(a) requires that a firm that continues to charge the high price observes a decrease in its demand when its competitor unilaterally marks the price down and observes an increase in its demand when its competitor runs out of stock. Assumption 1(b) requires that a firm that unilaterally marks the price down or whose competitor runs out of stock observes an increase in its demand. In general, Assumption 1(a) and (b) are satisfied if the two products offered by the firms are substitutes for one another. If the products are neither substitutable nor complementary or if the firms operate in two isolated markets, we have $\lambda_{F}=\lambda_{1}=\lambda_{M 1}$ and $\lambda_{2}=\lambda_{L}=\lambda_{M 2}$. Notice that $\lambda_{1}-\lambda_{F}$ measures the increase in the demand rate of a firm which charges $p_{1}$ when its competitor decreases the price from $p_{1}$ to $p_{2}$. Similarly, $\lambda_{L}-\lambda_{2}$ measures the decrease in the demand rate of a firm which charges $p_{2}$ when its competitor decreases the price from $p_{1}$ to $p_{2}$. In essence, these quantities measure how sensitive a firm's customers are to its competitor's prices. $\lambda_{M 1}-\lambda_{1}$ is the increase in the demand rate of a firm which charges $p_{1}$ when its competitor runs out of stock while charging $p_{1}$. Similarly, $\lambda_{M 2}-\lambda_{2}$ is the increase in the

Table 1

Demand rates for two competing firms.

\begin{tabular}{lllll}
\hline & & & & \\
\cline { 3 - 4 } & & $p_{1}$ & $p_{2}$ & sirm $B$ \\
& & $\lambda_{1}, \lambda_{1}$ & $\lambda_{F}, \lambda_{L}$ & $\lambda_{M 1}, 0$ \\
\multirow{3}{*}{ Firm $A$} & $p_{1}$ & $\lambda_{L}, \lambda_{F}$ & $\lambda_{2}, \lambda_{2}$ & $\lambda_{M 2}, 0$ \\
& $p_{2}$ & $0, \lambda_{M 1}$ & $0, \lambda_{M 2}$ & 0,0 \\
\hline
\end{tabular}


demand rate of a firm which charges $p_{2}$ when its competitor runs out of stock while charging $p_{2}$. These quantities measure how sensitive a firm's customers are to its competitor's stock situation. As the products are less differentiated or as demand interaction between firms increases we expect the differences $\lambda_{1}-\lambda_{F}, \lambda_{M 1}-\lambda_{1}, \lambda_{L}-\lambda_{2}$ and $\lambda_{M 2}-\lambda_{2}$ to get larger. Assumption 1 (c) requires that an industry-wide markdown increases the demand in both firms. This assumption is satisfied for all products with downward-sloping demand curves.

Assumption 2. The prices and demand rates satisfy

(a) $p_{2} \lambda_{L} \geq p_{1} \lambda_{1}$

(b) $p_{2} \lambda_{2} \geq p_{1} \lambda_{F}$

(c) $p_{2} \lambda_{M 2} \geq p_{1} \lambda_{M 1}$.

Assumption 2 ensures that any unilateral markdown, regardless of the competitor's stock situation or price, is increasing the instantaneous revenue rate for that firm (for otherwise, firms will never mark their prices down). A similar assumption, that a firm's revenue rate is decreasing in its own price, is also made in [7]. We only assume that this also holds for the duopoly case provided that the competitor's price and stock-out situation remains unchanged. In general, Assumption 2 is satisfied if a firm's own-price (arc) elasticity of demand is greater than or equal to 1 . For a firm that changes price from $p_{1}$ to $p_{2}$ while its competitor remains in stock with price $p_{1}$, own-price (arc) elasticity is defined as the percentage change in demand $\frac{\lambda_{L}-\lambda_{1}}{\frac{1}{2}\left(\lambda_{L}+\lambda_{1}\right)}$ divided by the percentage change in price $\frac{p_{1}-p_{2}}{\frac{1}{2}\left(p_{1}+p_{2}\right)}$ which is equal to $\frac{\left(p_{1} \lambda_{L}-p_{2} \lambda_{1}\right)+\left(p_{2} \lambda_{L}-p_{1} \lambda_{1}\right)}{\left(p_{1} \lambda_{L}-p_{2} \lambda_{1}\right)-\left(p_{2} \lambda_{L}-p_{1} \lambda_{1}\right)}$. This quantity is larger than or equal to 1 if and only if $p_{2} \lambda_{L}-p_{1} \lambda_{1} \geq 0$. Similarly, $p_{2} \lambda_{2}-p_{1} \lambda_{F} \geq 0$ is equivalent to a firm's own-price (arc) elasticity being larger than or equal to 1 when it changes the price from $p_{1}$ to $p_{2}$ while its competitor's price remains at $p_{2}$. Finally, $p_{2} \lambda_{M 2}-p_{1} \lambda_{M 1} \geq 0$ is equivalent to a firm's own-price (arc) elasticity being larger than or equal to 1 when it changes the price from $p_{1}$ to $p_{2}$ while its competitor remains out of stock. In the retail context, Assumption 2 requires that a firm that decreases its price observes an increase in its daily revenues if its competitor continues to (a) sell its product at the high price; (b) sell its product at the low price; (c) be out-of-stock. Notice that we do not require $p_{2} \lambda_{2} \geq p_{1} \lambda_{1}$; a markdown may or may not increase the revenue rate once it is matched by the competing firm.

The demand rates shown in Table 1 may result from an arbitrary demand model, with only mild restrictions provided in Assumptions 1 and 2 . We now present two specific, commonly used demand models and show how the demand rates in Table 1 can be specified using them.

\subsection{Linear demand model}

Linear demand models have been widely used in the marketing and economics literature to describe the relationship between demand and price (see [42], for example, for a model of demand in mixed retail and e-tail channels). We follow Shapley and Shubik [30] and represent the market as an aggregate consumer who maximizes the quadratic utility function

$U=a\left(\lambda^{A}+\lambda^{B}\right)-\frac{b}{2}\left(\lambda^{A}+\lambda^{B}\right)^{2}-\frac{\varepsilon}{4}\left(\lambda^{A}-\lambda^{B}\right)^{2}-p^{A} \lambda^{A}-p^{B} \lambda^{B}$,

where $\lambda^{A}$ and $\lambda^{B}$ are the demands (rate), $p^{A}$ and $p^{B}$ are the prices for firms $A$ and $B$, respectively. The parameter $\varepsilon(0<\varepsilon \leq 2 b)$ controls the degree of product differentiation. If it is close to zero, two products are close to perfect substitutes. If it is equal to $2 b$, they are unrelated. Solving the first order conditions $\left(\frac{\partial U}{\partial \lambda^{A}}=0\right.$ and $\left.\frac{\partial U}{\partial \lambda^{B}}=0\right)$, we recover the linear demand model for two products

$$
\begin{aligned}
\lambda^{A}\left(p^{A}, p^{B}\right)= & \frac{2 a-\left(1+\frac{2 b}{\varepsilon}\right) p^{A}-\left(1-\frac{2 b}{\varepsilon}\right) p^{B}}{4 b}, \text { and } \lambda^{B}\left(p^{A}, p^{B}\right) \\
& =\frac{2 a-\left(1-\frac{2 b}{\varepsilon}\right) p^{A}-\left(1+\frac{2 b}{\varepsilon}\right) p^{B}}{4 b} .
\end{aligned}
$$

In order to ensure that the products have non-negative demand at given prices, we need

$\varepsilon \geq \frac{2 b\left|p^{A}-p^{B}\right|}{2 a-p^{A}-p^{B}}$

Using (2), we get

$\lambda_{1}=\frac{a-p_{1}}{2 b}, \quad \lambda_{2}=\frac{a-p_{2}}{2 b}, \quad \lambda_{L}=\frac{2 a-\left(p_{1}+p_{2}\right)+\left(p_{1}-p_{2}\right) \frac{2 b}{\varepsilon}}{4 b}$,

and $\lambda_{F}=\frac{2 a-\left(p_{1}+p_{2}\right)-\left(p_{1}-p_{2}\right) \frac{2 b}{\varepsilon}}{4 b}$.

Maximizing the utility in (1) subject to constraint $\lambda^{B}=0$, we can get the demand rate of product $A$ when its price is $p^{A}$ and product $B$ is out of stock. Using this approach, we obtain the following demand rates:

$\lambda_{M 1}=\frac{a-p_{1}}{b+\frac{1}{2} \varepsilon}, \quad$ and $\quad \lambda_{M 2}=\frac{a-p_{2}}{b+\frac{1}{2} \varepsilon}$.

Note that finding $\lambda_{M 1}$ and $\lambda_{M 2}$ using this approach is equivalent to setting the price of the out-of-stock competitor to the maximum price that would satisfy the condition (3). 


\subsection{Attraction demand model}

The attraction demand model is a generalization of the logit demand model and is recently receiving more attention in economics and marketing literature. In this model, if firm $A$ charges $p^{A}$ and firm $B$ charges $p^{B}$, the purchase probability of firm $i$ 's product is given by

$\lambda^{A}\left(p^{A}, p^{B}\right)=\frac{a^{A}\left(p^{A}\right)}{a^{A}\left(p^{A}\right)+a^{B}\left(p^{B}\right)+\kappa}$

where $a^{i}($.$) is called the attraction function for firm i$ and $\kappa$ is a factor that accounts for the no-purchase option. It is assumed that $a^{i}($.$) is a$ positive and strictly decreasing function and $0 \leq \kappa \leq 1$.

Since we assume identical firms, we have $a^{A}()=.a^{B}()=.a\left(\right.$.). Denote $a_{1}=a\left(p_{1}\right)$ and $a_{2}=a\left(p_{2}\right)$. Clearly, if $p_{1} \geq p_{2}$, then $a_{2} \geq a_{1}$. Then, if customer arrival rate is $S$, we have:

$\lambda_{1}=\frac{S a_{1}}{2 a_{1}+\kappa}, \quad \lambda_{2}=\frac{S a_{2}}{2 a_{2}+\kappa}, \quad \lambda_{F}=\frac{S a_{1}}{a_{1}+a_{2}+\kappa}, \quad \lambda_{L}=\frac{S a_{2}}{a_{1}+a_{2}+\kappa}, \quad \lambda_{M 1}=\frac{S a_{1}}{a_{1}+\kappa}, \quad \lambda_{M 2}=\frac{S a_{2}}{a_{2}+\kappa}$.

While the firms are symmetric in market power, they may differ in forecasts or costs leading to ordering different amounts prior to the horizon. This leads to an asymmetry in starting inventory. At the beginning of horizon, we assume that firm $A$ is endowed with $n^{A}$ units of inventory and firm $B$ is endowed with $n^{B}$ units of inventory.

The problem for firms $A$ and $B$ is to find the price switch times $s^{A}$ and $s^{B}$, respectively, such that their revenues over the entire selling season are maximized. As in all simple timing games, each player's only choice is when to stop (markdown in our game).

We assume that the initial inventory levels are common knowledge. Initially, we assume that the firms pre-commit themselves to switch times at the beginning of the horizon. In other words, we consider open-loop strategies, and equilibrium in these strategies which is called open-loop equilibrium. This can be justified if the information lags are long and firms cannot observe and respond to their competitors' actions. Observability may be a problem in some revenue management industries [32]. Pre-commitment can also be partially justified on the grounds that firms may take time to prepare (advertising, store reorganization, moving inventory, etc.) for a markdown event. Some of the related literature also uses static games or open-loop equilibrium to study strategic interactions in revenue management competition, even in the presence of uncertain demand (e.g., [22,26]). In Section 6, we relax the pre-commitment assumption for an important subset of the parameter space and consider closed-loop equilibrium.

Before we characterize the equilibrium for the duopoly model, it may be useful to present the results for the case of a monopoly. Consider a firm that faces the problem of selling a fixed stock of $n$ units (we drop the index for the firms) over a horizon of length $t$. The starting price is $p_{1}$ which generates a demand rate of $\lambda_{1}$. The problem is to find the switch time $s$ after which the price is $p_{2}$ and the demand rate is $\lambda_{2}$. The objective of the firm is to maximize its total revenue which is

$\Phi(s)=p_{1} \min \left\{n, \lambda_{1} s\right\}+p_{2} \min \left\{\left(n-\lambda_{1} s\right)^{+}, \lambda_{2}(t-s)\right\}$.

The optimal switch time and the revenue can then be characterized easily as follows (which is simply a restatement of Proposition 4 for two prices in [11]).

Proposition 1. The optimal switch time and the optimal revenue for a monopoly firm are given by,

$\mathbf{s}= \begin{cases}0, & \text { if } n \geq \lambda_{2} t \text { and } p_{2} \lambda_{2}>p_{1} \lambda_{1} \\ \frac{\lambda_{2} t-n}{\lambda_{2}-\lambda_{1}}, & \text { if } \lambda_{1} t<n<\lambda_{2} t \text { and } p_{2} \lambda_{2}>p_{1} \lambda_{1} \\ t, & \text { if } n \leq \lambda_{1} t \text { or } p_{2} \lambda_{2} \leq p_{1} \lambda_{1},\end{cases}$

$\mathbf{\Phi}=p_{1} \lambda_{1} \mathbf{s}+p_{2} \lambda_{2}(t-\mathbf{s})$

Note first that in order for the firm to have an incentive to change its price, revenue rate should be increasing with the price change, i.e., $p_{1} \lambda_{1}<p_{2} \lambda_{2}$. In addition, the firm should not be able to finish off its inventory with the initial price alone, i.e., $n>\lambda_{1} t$. If one of these conditions is not satisfied, the firm will not change the price $(\mathbf{s}=t)$. With the exclusion of these trivial cases, the firm will change the price at the latest possible time that it can still sell all of its inventory. If the horizon is not long enough or the demand rate for the low price is not high enough to finish the inventory $\left(n>\lambda_{2} t\right)$, the firm changes the price at the beginning of the selling season $(\mathbf{s}=0)$. Using $(6)$ and $(7)$, one can easily establish the following monotonicity results: (i) $\mathbf{s}$ is decreasing in $n$ and increasing in $t$, and, (ii) $\boldsymbol{\Phi}$ is increasing in $n$ and $t$ (throughout the paper, we use the words increasing and decreasing in a non-strict sense).

\section{Equilibrium}

When the demand for each firm depends on its competitor's price and stock-out situation as described in Table 1, firms can no longer optimize their profits by only considering their own actions. We use a game theoretic model to find the equilibrium price switch times. We use a non-cooperative game with complete information. That is, the rules of the game are common knowledge. Each firm knows its own and its competitor's starting inventory levels, demand rates and payoff functions, as well as the length of the selling season. 
When open-loop strategies are considered, each firm $i$ 's strategy is its price switch time, denoted by $s^{i} \in[0, t]$ which it commits to at the beginning of the horizon. For a given set of price switch times, the payoff for firm $A$ can be written as follows:

$$
\Phi^{A}\left(s^{A}, s^{B}\right)= \begin{cases}\Phi_{1}^{A}\left(s^{A}, s^{B}\right), & \text { if } s^{A} \leq \min \left\{s^{B}, \eta_{5}\right\} \text { and } \lambda_{1} s^{A}+\lambda_{F}\left(s^{B}-s^{A}\right)+\lambda_{2}\left(t-s^{B}\right) \leq n^{B} \\ \Phi_{2}^{A}\left(s^{A}, s^{B}\right), & \text { if } s^{A} \leq \min \left\{s^{B}, \eta_{5}\right\} \text { and } \lambda_{1} s^{A}+\lambda_{F}\left(s^{B}-s^{A}\right)+\lambda_{2}\left(t-s^{B}\right)>n^{B} \geq \lambda_{1} s^{A}+\lambda_{F}\left(s^{B}-s^{A}\right) \\ \Phi_{3}^{A}\left(s^{A}, s^{B}\right), & \text { if } s^{A} \leq \min \left\{s^{B}, \eta_{5}\right\} \text { and } \lambda_{1} s^{A}+\lambda_{F}\left(s^{B}-s^{A}\right)>n^{B} \\ \Phi_{4}^{A}\left(s^{A}, s^{B}\right), & \text { if } s^{A}>s^{B} \text { and } \lambda_{1} s^{B}+\lambda_{L}\left(s^{A}-s^{B}\right)+\lambda_{2}\left(t-s^{A}\right) \leq n^{B} \\ \Phi_{5}^{A}\left(s^{A}, s^{B}\right), & \text { if } s^{A}>s^{B} \text { and } \lambda_{1} s^{B}+\lambda_{L}\left(s^{A}-s^{B}\right)+\lambda_{2}\left(t-s^{A}\right)>n^{B} \geq \lambda_{1} s^{B}+\lambda_{L}\left(s^{A}-s^{B}\right) \\ \Phi_{6}^{A}\left(s^{A}, s^{B}\right), & \text { if } s^{A}>s^{B} \text { and } \lambda_{1} s^{B}+\lambda_{L}\left(s^{A}-s^{B}\right)>n^{B} \geq \lambda_{1} s^{B} \\ \Phi_{7}^{A}\left(s^{A}, s^{B}\right), & \text { otherwise, }\end{cases}
$$

where

$$
\begin{aligned}
& \Phi_{1}^{A}\left(s^{A}, s^{B}\right)=p_{1} \min \left\{n^{A}, \lambda_{1} s^{A}\right\}+p_{2} \min \left\{\left(n^{A}-\lambda_{1} s^{A}\right)^{+}, \lambda_{L}\left(s^{B}-s^{A}\right)+\lambda_{2}\left(t-s^{B}\right)\right\} \\
& \Phi_{2}^{A}\left(s^{A}, s^{B}\right)=p_{1} \min \left\{n^{A}, \lambda_{1} s^{A}\right\}+p_{2} \min \left\{\left(n^{A}-\lambda_{1} s^{A}\right)^{+}, \lambda_{L}\left(s^{B}-s^{A}\right)+\lambda_{2}\left(\eta_{1}-s^{B}\right)+\lambda_{M 2}\left(t-\eta_{1}\right)\right\} \\
& \Phi_{3}^{A}\left(s^{A}, s^{B}\right)=p_{1} \min \left\{n^{A}, \lambda_{1} s^{A}\right\}+p_{2} \min \left\{\left(n^{A}-\lambda_{1} s^{A}\right)^{+}, \lambda_{L}\left(\eta_{2}-s^{A}\right)+\lambda_{M 2}\left(t-\eta_{2}\right)\right\} \\
& \Phi_{4}^{A}\left(s^{A}, s^{B}\right)=p_{1} \min \left\{n^{A}, \lambda_{1} s^{B}+\lambda_{F}\left(s^{A}-s^{B}\right)\right\}+p_{2} \min \left\{\left(n^{A}-\lambda_{1} s^{B}-\lambda_{F}\left(s^{A}-s^{B}\right)\right)^{+}, \lambda_{2}\left(t-s^{A}\right)\right\} \\
& \Phi_{5}^{A}\left(s^{A}, s^{B}\right)=p_{1} \min \left\{n^{A}, \lambda_{1} s^{B}+\lambda_{F}\left(s^{A}-s^{B}\right)\right\}+p_{2} \min \left\{\left(n^{A}-\lambda_{1} s^{B}-\lambda_{F}\left(s^{A}-s^{B}\right)\right)^{+}, \lambda_{2}\left(\eta_{3}-s^{A}\right)+\lambda_{M 2}\left(t-\eta_{3}\right)\right\}, \\
& \Phi_{6}^{A}\left(s^{A}, s^{B}\right)=p_{1} \min \left\{n^{A}, \lambda_{1} s^{B}+\lambda_{F}\left(\eta_{4}-s^{B}\right)+\lambda_{M 1}\left(s^{A}-\eta_{4}\right)\right\}+p_{2} \min \left\{\left(n^{A}-\lambda_{1} s^{B}-\lambda_{F}\left(\eta_{4}-s^{B}\right)-\lambda_{M 1}\left(s^{A}-\eta_{4}\right)\right)^{+}, \lambda_{M 2}\left(t-s^{A}\right)\right\} \\
& \Phi_{7}^{A}\left(s^{A}, s^{B}\right)=p_{1} \min \left\{n^{A}, \lambda_{1} \eta_{5}+\lambda_{M 1}\left(s^{A}-\eta_{5}\right)\right\}+p_{2} \min \left\{\left(n^{A}-\lambda_{1} \eta_{5}-\lambda_{M 1}\left(s^{A}-\eta_{5}\right)\right)^{+}, \lambda_{M 2}\left(t-s^{A}\right)\right\}
\end{aligned}
$$

and $\eta_{1}, \eta_{2}, \eta_{3}, \eta_{4}$ and $\eta_{5}$ are solutions to

$$
\lambda_{1} s^{A}+\lambda_{F}\left(s^{B}-s^{A}\right)+\lambda_{2}\left(\eta_{1}-s^{B}\right)=n^{B}, \lambda_{1} s^{A}+\lambda_{F}\left(\eta_{2}-s^{A}\right)=n^{B}, \lambda_{1} s^{B}+\lambda_{L}\left(s^{A}-s^{B}\right)+\lambda_{2}\left(\eta_{3}-s^{A}\right)=n^{B}, \lambda_{1} s^{B}+\lambda_{L}\left(\eta_{4}-s^{B}\right)=n^{B}, \lambda_{1} \eta_{5}=n^{B} .
$$

The seven conditions in (8) correspond to the following cases. In case 1, firm $A$ switches before firm $B$ and firm $B$ does not run out of stock during the horizon. In case 2, firm $A$ switches before firm $B$ and firm $B$ runs out of stock after firm $A$ switches and before the end of the horizon at time $\eta_{1}$. In case 3 , firm $A$ switches and firm $B$ runs out of stock at time $\eta_{4}$ before it switches. In case 4 , firm $B$ switches after firm $A$ and does not run out of stock during the horizon. In case 5 , firm $B$ switches before firm $A$, but runs out of stock after firm $A$ switches and before the end of the horizon at time $\eta_{3}$. In case 6 , firm $B$ switches before firm $A$, but runs out of stock before firm $A$ switches at time $\eta_{4}$. In case 7, firm $B$ runs out of stock at time $\eta_{5}$ before it switches. The payoff function $\Phi^{B}\left(s^{A}, s^{B}\right)$ for firm $B$ can be similarly defined.

We now characterize the open-loop equilibrium for the markdown timing game, where firms' payoffs, as a function of their own and competitor's switch times, are described in (8). For the remainder of the analysis, we assume, without loss of generality, that $n^{A} \geq n^{B}$, i.e., firm $A$ is the larger firm and firm $B$ is the smaller firm.

The existence and characterization of the pure-strategy equilibrium critically depend on the value of $\lambda_{M 2}$, the demand rate of a firm when it charges the low price $p_{2}$ and its competitor is out of stock. Our analysis will show that some of the equilibria involve delaying the markdown time so as to force the other firm run out of inventory and enjoy monopoly demand rates $\lambda_{M 1}$ or $\lambda_{M 2}$. Observe also that $\lambda_{M 2}$ is the largest demand rate that a single firm can get as a monopoly and the value of $\lambda_{M 1}$ is bounded from above by $p_{2} \lambda_{M 2} / p_{1}$ due to Assumption 2(c). Therefore, the magnitude of $\lambda_{M 2}$ in comparison to demand rates when the competitor is in stock $\left(\lambda_{1}, \lambda_{2}, \lambda_{F}, \lambda_{L}\right)$ plays an instrumental role in determining which of the equilibria will be played in the markdown competition game.

The following three thresholds on $\lambda_{M 2}$ are necessary to identify the equilibrium:

$\chi_{1}=\frac{\lambda_{2}\left(p_{2} \lambda_{L}-p_{1} \lambda_{F}\right)}{p_{2}\left(\lambda_{L}-\lambda_{2}\right)}=\lambda_{2}\left(1+\frac{p_{2} \lambda_{2}-p_{1} \lambda_{F}}{p_{2}\left(\lambda_{L}-\lambda_{2}\right)}\right)$

$\chi_{2}=\frac{\lambda_{2}\left(p_{2} \lambda_{L}-p_{1} \lambda_{1}+p_{2}\left(\lambda_{1}-\lambda_{F}\right)\right)}{p_{2}\left(\lambda_{1}-\lambda_{F}\right)}=\lambda_{2}\left(1+\frac{p_{2} \lambda_{L}-p_{1} \lambda_{1}}{p_{2}\left(\lambda_{1}-\lambda_{F}\right)}\right)$,

$\chi_{3}=\frac{\lambda_{1}\left(p_{2} \lambda_{L}-p_{1} \lambda_{F}\right)}{p_{2}\left(\lambda_{1}-\lambda_{F}\right)}=\lambda_{1}\left(\frac{p_{1}}{p_{2}}+\frac{p_{2} \lambda_{L}-p_{1} \lambda_{1}}{p_{2}\left(\lambda_{1}-\lambda_{F}\right)}\right)$.

Note that $2 \lambda_{2}$ is an absolute upper bound on $\lambda_{M 2}$. Since we also have $p_{2} \lambda_{2} \geq p_{1} \lambda_{F}$ and $p_{2} \lambda_{L} \geq p_{1} \lambda_{1}$, using (9) and (10) we obtain $\lambda_{M 2} \leq \max \left\{\chi_{1}, \chi_{2}\right\}$, i.e., $\lambda_{M 2}$ cannot be larger than both $\chi_{1}$ and $\chi_{2}$.

We can show that the conditions $\lambda_{M 2} \leq \chi_{i}, i=1,2,3$ are equivalent to the following conditions:

$\frac{p_{2} \lambda_{M 2}-p_{2} \lambda_{2}}{\lambda_{2}} \leq \frac{p_{2} \lambda_{2}-p_{1} \lambda_{F}}{\lambda_{L}-\lambda_{2}}$

$\frac{p_{2} \lambda_{M 2}-p_{2} \lambda_{2}}{\lambda_{2}} \leq \frac{p_{2} \lambda_{L}-p_{1} \lambda_{1}}{\lambda_{1}-\lambda_{F}}$ 
$\frac{p_{2} \lambda_{M 2}-p_{1} \lambda_{1}}{\lambda_{1}} \leq \frac{p_{2} \lambda_{L}-p_{1} \lambda_{1}}{\lambda_{1}-\lambda_{F}}$

We have already seen in Section 3 that the quantities $p_{2} \lambda_{2}-p_{1} \lambda_{F}$ and $p_{2} \lambda_{L}-p_{1} \lambda_{1}$ represent increases in revenue rates due to a (own) markdown and are related to own-price elasticity of demand. Similarly, the quantity $p_{2} \lambda_{M 2}-p_{2} \lambda_{2}$ is the increase in the revenue rate of a firm whose competitor runs out of stock and can be considered as a measure of how elastic a firm's demand is to its competitor's stockout. Finally, the quantity $p_{2} \lambda_{M 2}-p_{1} \lambda_{1}=\left(p_{2} \lambda_{M 2}-p_{1} \lambda_{M 1}\right)+\left(p_{1} \lambda_{M 1}-p_{1} \lambda_{1}\right)$ captures the simultaneous effect of reducing (own) price and the competitor running out-of-stock on the revenue rate of a firm.

Given these definitions, the left-hand sides of (12) and (13) measures the elasticity of a firm's demand to its competitor's stock-out per unit of decrease in competitor's demand rate. The right-hand sides of (12) and (13) can be considered as the elasticities of a firm's demand to its own price drop, while the competitor continues to charge $p_{2}$ and $p_{1}$, respectively, adjusted by its competitor's demand rate decrease. Given these definitions, conditions (12) and (13) make executing the markdowns when the competitor is still in stock more attractive. This ensures that forcing the rival firm to run out of stock and subsequently monopolizing the total demand is not an equilibrium strategy for a firm (unless this ensures exhausting inventory for that firm). We will see in Section 4.3 that when these two conditions are not satisfied, there is no equilibrium in the markdown timing game in pure strategies. These conditions in spirit are similar to a required upper bound on the substitution elasticity for the existence of pure-strategy equilibria in Bertrand-Edgeworth-Chamberlin competition shown by Benassy [4]. In this case, we require bounds on elasticity of one firm's demand to its competitor's stock-out since stock-outs are the sources of instability in our game.

The left-hand side of (14) measures the elasticity of a firm's demand to simultaneous events of competitor running out of stock and (own) price reduction scaled by the change in demand rate of the competing firm. Therefore, the condition (14) ensures that a firm does not have the incentive to delay its markdown until its competitor runs out of stock unless it can exhaust its inventory.

\subsection{Equilibrium characterization for $\lambda_{M 2} \leq \min \left\{\chi_{1}, \chi_{2}, \chi_{3}\right\}$}

Equilibrium switch times are obviously functions of the parameters of the game. When we assume that $\lambda_{M 2} \leq \min \left\{\chi_{1}, \chi_{2}, \chi_{3}\right\}$, we show that the equilibrium switch times depend on $\lambda_{1}, \lambda_{2}, \lambda_{F}, \lambda_{L}, \lambda_{M 1}, \lambda_{M 2}, n^{A}, n^{B}$ and $t$ (given $p_{1}$ and $p_{2}$ ). Equilibrium behavior depends on where the actual parameters of the game falls in the parameter space. (Note however that the demand rates are functions of the initial price $p_{1}$ and markdown price $p_{2}$ as exemplified in Section 3. Therefore, these prices also affect which of these equilibria will be played). Before we formally characterize the equilibrium in Theorem 1, we describe the equilibrium behavior under different regions of the parameter space. We identify seven regions, where the equilibrium behavior is qualitatively different in each. These regions and the corresponding equilibrium behavior are given in Table 2 .

Note that in regions II, IV, V and VI, one or both firms switch after the season starts. In all these cases, the firm(s) which switches inside the season switch at such a time that it depletes its inventory precisely when the season ends. In regions II, IV and V, the firm which switches the price down does so when its competitor is still in stock. Only in region VI, one firm marks the price down when its competitor is out of stock. In this case, firm $A$ monopolizes demand after firm $B$ runs out of inventory. This case is similar to what is described as buffering in Ghemawat and McGahan [13]. In regions I, III and VII, switching inside the season is not an equilibrium behavior for neither firm; both firms either switch at the start of the season, or never switch.

In order to describe regions I-VII, we need to define the following thresholds, which we will compare against the season length $(t)$.

$X_{1}=\frac{n^{B}}{\lambda_{2}}$

$X_{2}=\frac{n^{B}}{\lambda_{F}}$

$X_{3}=\frac{n^{B}}{\lambda_{2}}+\frac{\left(\lambda_{2}-\lambda_{F}\right)\left(n^{A}-n^{B}\right)}{\lambda_{2}\left(\lambda_{L}-\lambda_{F}\right)}=\frac{\left(\lambda_{2}-\lambda_{F}\right) n^{A}}{\lambda_{2}\left(\lambda_{L}-\lambda_{F}\right)}+\frac{\left(\lambda_{L}-\lambda_{2}\right) n^{B}}{\lambda_{2}\left(\lambda_{L}-\lambda_{F}\right)}$,

$X_{4}=\frac{n^{B}}{\lambda_{1}}+\frac{\left(\lambda_{1}-\lambda_{F}\right)\left(n^{A}-n^{B}\right)}{\lambda_{1}\left(\lambda_{L}-\lambda_{F}\right)}=\frac{\left(\lambda_{1}-\lambda_{F}\right) n^{A}}{\lambda_{1}\left(\lambda_{L}-\lambda_{F}\right)}+\frac{\left(\lambda_{L}-\lambda_{1}\right) n^{B}}{\lambda_{1}\left(\lambda_{L}-\lambda_{F}\right)}$,

$X_{5}=\frac{n^{B}}{\lambda_{F}}+\frac{\left(\lambda_{F} n^{A}-\lambda_{L} n^{B}\right)}{\lambda_{F} \lambda_{M 2}}=\frac{n^{A}}{\lambda_{M 2}}+\frac{\left(\lambda_{M 2}-\lambda_{L}\right) n^{B}}{\lambda_{F} \lambda_{M 2}}$,

$X_{6}=\frac{n^{B}}{\lambda_{1}}+\frac{\left(n^{A}-n^{B}\right)}{\lambda_{M 2}}=\frac{n^{A}}{\lambda_{M 2}}+\frac{\left(\lambda_{M 2}-\lambda_{1}\right) n^{B}}{\lambda_{1} \lambda_{M 2}}$,

$X_{7}=\frac{n^{B}}{\lambda_{1}}+\frac{\left(n^{A}-n^{B}\right)}{\lambda_{M 1}}=\frac{n^{A}}{\lambda_{M 1}}+\frac{\left(\lambda_{M 1}-\lambda_{1}\right) n^{B}}{\lambda_{1} \lambda_{M 1}}$.

We are now ready to formally state our result for the equilibrium of the game (all proofs are relegated to Appendix). 
Table 2

Equilibrium behavior when $\lambda_{M 2} \leq \min \left\{\chi_{1}, \chi_{2}, \chi_{3}\right\}$.

\begin{tabular}{ll}
\hline Region & Equilibrium behavior \\
\hline I & Both firms switch at time 0 \\
II & Firm A switches at time 0, firm B switches in $(0, t)$ \\
III & Firm A switches at time 0, firm B does not switch \\
IV & Both firms switch in $(0, t)$, firm A switches before firm B \\
V & Firm B does not switch, firm A switches before firm B runs out of stock \\
VI & Firm B does not switch, firm A switches after firm B runs out of stock \\
VII & Neither firm switches \\
\hline
\end{tabular}

Theorem 1. The following is the pure-strategy Nash equilibrium of the markdown timing game if $\lambda_{M 2} \leq \min \left\{\chi_{1}, \chi_{2}, \chi_{3}\right\}$,

$$
\left(\mathbf{s}^{A}, \mathbf{s}^{B}\right)= \begin{cases}(0,0), & \text { if } t \leq X_{1}, \\ \left(0, \frac{\lambda_{2} t-n^{B}}{\lambda_{2}-\lambda_{F}}\right), & \text { if } X_{1}<t \leq \min \left\{X_{2}, X_{3}\right\}, \quad \text { (II) } \\ (0, t), & \text { if } X_{2}<t \leq X_{5}, \\ \left(\frac{\lambda_{2}\left(\lambda_{L}-\lambda_{F}\right) t-\left(\lambda_{2}-\lambda_{F}\right) n^{A}-\left(\lambda_{L}-\lambda_{2}\right) n^{B}}{\left(\lambda_{2}-\lambda_{1}\right)\left(\lambda_{L}-\lambda_{F}\right)}, \frac{\lambda_{2}\left(\lambda_{L}-\lambda_{F}\right) t-\left(\lambda_{1}-\lambda_{F}\right) n^{A}-\left(\lambda_{L}-\lambda_{1}\right) n^{B}}{\left(\lambda_{2}-\lambda_{1}\right)\left(\lambda_{L}-\lambda_{F}\right)}\right), & \text { if } X_{3}<t \leq X_{4}, \\ \left(\frac{-\lambda_{F} \lambda_{M 2} t+\lambda_{F} n^{A}+\left(\lambda_{M 2}-\lambda_{L}\right) n^{B}}{\lambda_{M 2}\left(\lambda_{1}-\lambda_{F}\right)-\lambda_{1}\left(\lambda_{L}-\lambda_{F}\right)}, t\right), & \text { if } \max \left\{X_{4}, X_{5}\right\}<t \leq X_{6}, \quad \text { (IV) } \\ \left(\frac{\lambda_{M 2} \lambda_{1} t-\lambda_{1} n^{A}-\left(\lambda_{M 1}-\lambda_{1}\right) n^{B}}{\lambda_{1}\left(\lambda_{M 2}-\lambda_{M 1}\right)}, t\right), & \text { if } X_{6}<t \leq X_{7}, \quad \text { if } X_{7}<t . \\ (t, t), & \text { (VI) }\end{cases}
$$

Each case in Theorem 1 refers to the corresponding equilibrium behavior and region given in Table 2 . Note that these regions are stated as conditions on $t$ and $X_{i}$, where $X_{i}, i=1, \ldots, 7$ are functions of $n^{A}$ and $n^{B}$, as given in (15)-(21). Equilibrium payoffs for each region are given below:

$$
\left(\boldsymbol{\Phi}^{\mathbf{A}}, \boldsymbol{\Phi}^{\mathbf{B}}\right)=\left\{\begin{array}{l}
\left(p_{2} \lambda_{2} t, p_{2} \lambda_{2} t\right), \\
\left(\frac{p_{2} \lambda_{2}\left(\lambda_{L}-\lambda_{F}\right) t-p_{2}\left(\lambda_{L}-\lambda_{2}\right) n^{B}}{\lambda_{2}-\lambda_{F}}, \frac{\left(p_{1}-p_{2}\right) \lambda_{2} \lambda_{F} t+\left(p_{2} \lambda_{2}-p_{1} \lambda_{F}\right) n^{B}}{\lambda_{2}-\lambda_{F}}\right) \\
\left(\frac{p_{2} \lambda_{M 2} \lambda_{F} t-p_{2}\left(\lambda_{M 2}-\lambda_{L}\right) n^{B}}{\lambda_{F}}, p_{1} n^{B}\right), \\
\left(\frac{\left(p_{1}-p_{2}\right) \lambda_{1} \lambda_{2}\left(\lambda_{L}-\lambda_{F}\right) t+\left[\left(p_{2} \lambda_{L}-p_{1} \lambda_{1}\right)\left(\lambda_{2}-\lambda_{1}\right)+\left(p_{2} \lambda_{2}-p_{1} \lambda_{1}\right)\left(\lambda_{1}-\lambda_{F}\right)\right] n^{A}-\left(p_{1}-p_{2}\right) \lambda_{1}\left(\lambda_{L}-\lambda_{2}\right) n^{B}}{\left(\lambda_{2}-\lambda_{1}\right)\left(\lambda_{L}-\lambda_{F}\right)}\right. \\
\left.\frac{\left(p_{1}-p_{2}\right) \lambda_{1} \lambda_{2}\left(\lambda_{L}-\lambda_{F}\right) t-\left(p_{1}-p_{2}\right) \lambda_{2}\left(\lambda_{1}-\lambda_{F}\right) n^{A}+\left[\left(p_{2} \lambda_{2}-p_{1} \lambda_{F}\right)\left(\lambda_{2}-\lambda_{1}\right)+\left(p_{2} \lambda_{2}-p_{1} \lambda_{1}\right) 2\left(\lambda_{L}-\lambda_{2}\right)\right] n^{B}}{\left(\lambda_{2}-\lambda_{1}\right)\left(\lambda_{L}-\lambda_{F}\right)}\right) \\
\left(\frac{\left(p_{1}-p_{2}\right) \lambda_{1} \lambda_{F} \lambda_{M 2} t+\left[\lambda_{1}\left(p_{2} \lambda_{L}-p_{1} \lambda_{F}\right)-p_{2} \lambda_{M 2}\left(\lambda_{1}-\lambda_{F}\right)\right] n^{A}-\left(p_{1}-p_{2}\right) \lambda_{1}\left(\lambda_{M 2}-\lambda_{L}\right) n^{B}}{\lambda_{1}\left(\lambda_{L}-\lambda_{F}\right)-\lambda_{M 2}\left(\lambda_{1}-\lambda_{F}\right)}, p_{1} n^{B}\right) \\
\lambda_{1}\left(\lambda_{M 2}-\lambda_{M 1}\right) \\
\left(\frac{\left(p_{1}-p_{2}\right) \lambda_{1} \lambda_{M 1} \lambda_{M 2} t+\left(p_{2} \lambda_{M 2}-p_{1} \lambda_{M 1}\right) \lambda_{1} n^{A}-\left(p_{1}-p_{2}\right) \lambda_{M 2}\left(\lambda_{M 1}-\lambda_{1}\right) n^{B}}{\left.p_{1} n^{B}\right)},\right. \\
\left(p_{1} n^{A}, p_{1} n^{B}\right) .
\end{array}\right.
$$

If we fix demand rates and season length, we can represent each region in Table 2 in a two-dimensional plot of $n^{A}$ and $n^{B}$. One such graph is given in Fig. 1 for $\lambda_{1}=2 / 7, \lambda_{2}=4.5 / 7, \lambda_{F}=1 / 7, \lambda_{L}=5 / 7, \lambda_{M 1}=8 / 21, \lambda_{M 2}=16 / 21$ and $t=100$. A reflection of these regions with respect to the $45 \%$ line $\left(n^{A}=n^{B}\right)$ would give the complete characterization of the equilibrium behavior when we allow $n^{B}$ to be larger than $n^{A}$ (In this case, equilibrium in each mirror region will be defined similar to the original region except that firm $A$ is replaced with firm $B$ and vice versa).

The characterization provided in Theorem 1 leads to the following important qualitative results regarding the switch times in all possible equilibria.

Corollary 1. If $\lambda_{M 2} \leq \min \left\{\chi_{1}, \chi_{2}, \chi_{3}\right\}$, firm A marks its price down earlier than firm $B$ in equilibrium.

Without competition, it is obvious that the larger firm will have an earlier markdown time since it will need a longer time over which it has a higher demand rate to liquidate its inventory. Corollary 1 shows that this result continues to hold in the presence of competition, i.e., the smaller firm does not have any incentive to preempt the larger firm in markdown timing competition.

Corollary 2. If $\lambda_{M 2} \leq \min \left\{\chi_{1}, \chi_{2}, \chi_{3}\right\}$, in equilibrium, each firm follows one of three strategies:

(i) Mark the price down at the beginning of the season and sell as much inventory as possible at the low price.

(ii) Never mark the price down, and sell all inventory at the high price.

(iii) Mark the price down at such a time that all inventory is exhausted precisely at the end of the season. 


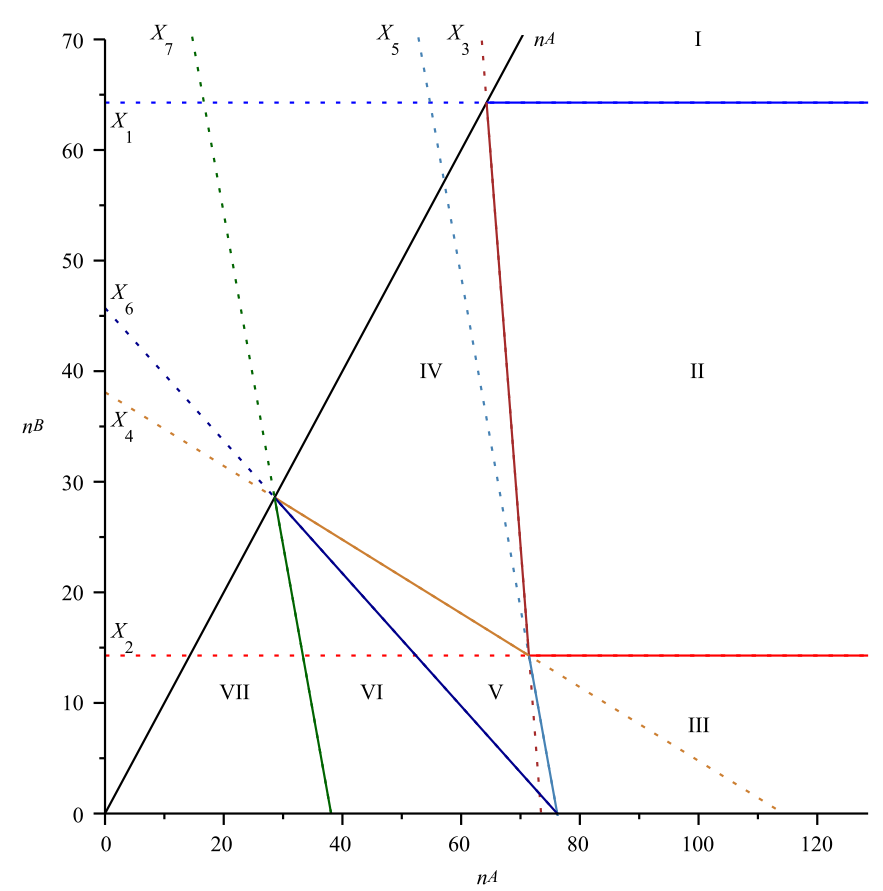

Fig. 1. Equilibrium regions when $\lambda_{M 2} \leq \min \left\{\chi_{1}, \chi_{2}, \chi_{3}\right\}$ for $\lambda_{1}=2 / 7, \lambda_{2}=4.5 / 7, \lambda_{F}=1 / 7, \lambda_{L}=5 / 7, \lambda_{M 1}=8 / 21, \lambda_{M 2}=16 / 21$ and $t=100$.

Table 3

Equilibrium behavior when $\chi_{3}<\lambda_{M 2} \leq \min \left\{\chi_{1}, \chi_{2}\right\}$.

\begin{tabular}{ll}
\hline Region & Equilibrium behavior \\
\hline I & Both firms switch at time 0 \\
II & Firm A switches at time 0, firm B switches in $(0, t)$ \\
IV & Both firms switch in $(0, t)$, firm A switches before firm B \\
VI & Firm B does not switch, firm A switches after firm B runs out of stock \\
VII & Neither firm switches \\
VIII & Firm B does not switch, firm A switches right when firm B runs out of \\
& stock \\
\hline
\end{tabular}

Corollary 2 states that firms will never select a markdown time inside the season that leads to leftover inventory at the end of the season or a stock-out before the season ends. In other words, when the benefits of having the rival firm run out of stock is not high (this may happen when firms operate in far-away markets or sell products that are differentiated), each firm will ensure that the markdown time it select will not lead to any leftover inventory except when it is clearly overstocked and mark its price down at the beginning of the season. Similarly, a firm should never select a markdown time that will lead to a stock-out for itself. Stock-outs will only happen when a firm is clearly understocked; a case in which the firm never marks the price down. The results show that in industries with no or relatively low uncertainty, strategic interactions between competing firms should not lead to leftover inventory or unsatisfied demand as long as the firms are not clearly overstocked or understocked (for example, when $\lambda_{1} t \leq n^{B} \leq n^{A} \leq \lambda_{2} t$; both firms can finish their inventory if they both use a low price and neither firm can finish inventory if both firms use the high price). This is true even when the firms have a single chance to change the price to a pre-set level.

\subsection{Equilibrium characterization for $\chi_{3}<\lambda_{M 2} \leq \min \left\{\chi_{1}, \chi_{2}\right\}$}

When we allow $\lambda_{M 2}$ to be larger than $\chi_{3}=\frac{\lambda_{1}\left(p_{2} \lambda_{L}-p_{1} \lambda_{F}\right)}{p_{2}\left(\lambda_{1}-\lambda_{F}\right)}$, equilibrium behavior changes in certain regions of the parameter space defined in Section 4.1. For the larger firm, forcing the competitor to run out of stock and marking the price down right at that instant becomes an equilibrium for a subset of the parameter space (now called region VIII). This can be considered an extreme form of buffering as the larger firm does this in the expense of having some inventory left at the end of the season. Also, the equilibria defined for regions III and $\mathrm{V}$ that are observed for the case $\lambda_{M 2} \leq \min \left\{\chi_{1}, \chi_{2}, \chi_{3}\right\}$ are no longer possible outcomes of the game. We define the regions and equilibrium behavior for this case in Table 3. While regions I, VII and VIII can be described by thresholds defined in (15)-(21), we need the following two new thresholds to revise the definitions of regions II and IV and to define region VIII:

$X_{2}^{b}=\frac{p_{2} \lambda_{1}\left(\lambda_{L}-\lambda_{2}\right)+p_{1} \lambda_{1}\left(\lambda_{2}-\lambda_{F}\right)-p_{2} \lambda_{M 2}\left(\lambda_{2}-\lambda_{F}\right)}{p_{2} \lambda_{1} \lambda_{2}\left(\lambda_{L}-\lambda_{F}\right)-p_{2} \lambda_{1} \lambda_{M 2}\left(\lambda_{2}-\lambda_{F}\right)} n^{B}$,

$X_{4}^{b}=\frac{n^{B}}{\lambda_{1}}+\left[\frac{p_{2} \lambda_{2}\left(\lambda_{L}-\lambda_{F}\right)-\lambda_{1}\left(p_{2} \lambda_{L}-p_{1} \lambda_{F}\right)-\left(p_{1}-p_{2}\right) \lambda_{1} \lambda_{2}}{p_{2} \lambda_{M 2}\left(\lambda_{2}-\lambda_{1}\right)-\left(p_{1}-p_{2}\right) \lambda_{1} \lambda_{2}}\right] \frac{\left(n^{A}-n^{B}\right)}{\left(\lambda_{L}-\lambda_{F}\right)}$.

The following theorem formally characterizes the equilibrium for the case $\chi_{3}<\lambda_{M 2} \leq \min \left\{\chi_{1}, \chi_{2}\right\}$ : 


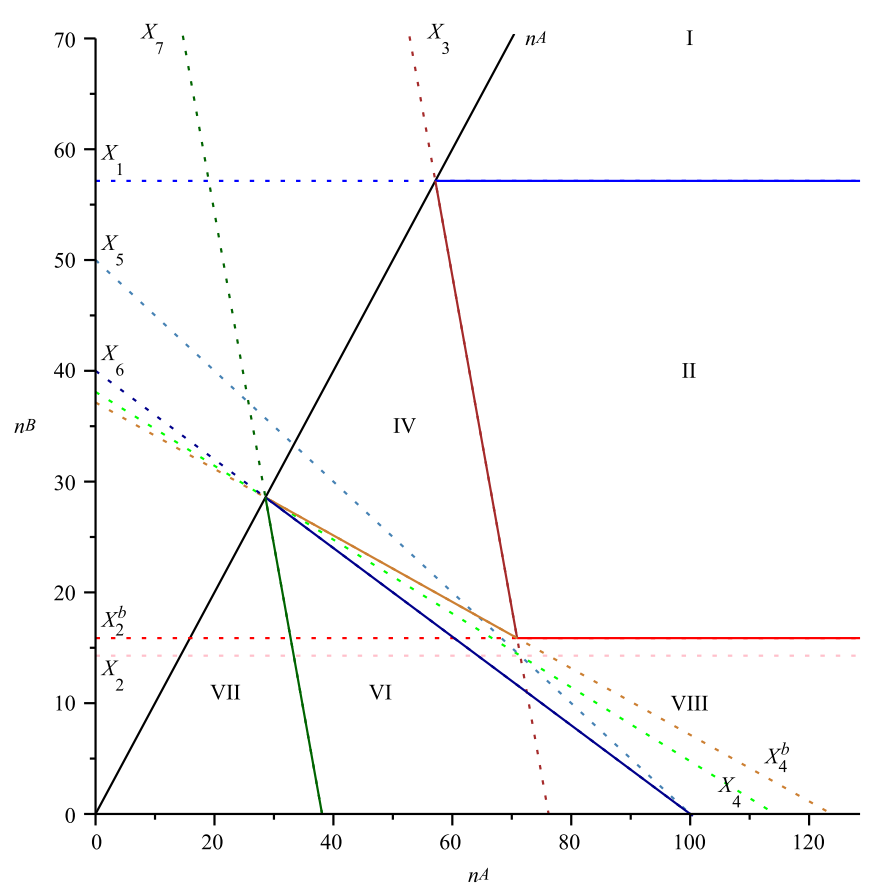

Fig. 2. Equilibrium regions when $\chi_{3}<\lambda_{M 2}<\min \left\{\chi_{1}, \chi_{2}\right\}$ for $\lambda_{1}=2 / 7, \lambda_{2}=4 / 7, \lambda_{F}=1 / 7, \lambda_{L}=5 / 7, \lambda_{M 1}=8 / 21, \lambda_{M 2}=1, p_{1}=10, p_{2}=6$ and $t=100$.

Theorem 2. The following is the pure-strategy Nash equilibrium of the markdown timing game if $\chi_{3}<\lambda_{M 2} \leq \min \left\{\chi_{1}, \chi_{2}\right\}$,

$$
\left(\mathbf{s}^{A}, \mathbf{s}^{B}\right)= \begin{cases}(0,0), & \text { if } t \leq X_{1}, \\ \left(0, \frac{\lambda_{2} t-n^{B}}{\lambda_{2}-\lambda_{F}}\right), & \text { if } X_{1}<t \leq \min \left\{X_{2}^{b}, X_{3}\right\}, \quad \text { (II) } \\ \left(\frac{\lambda_{2}\left(\lambda_{L}-\lambda_{F}\right) t-\left(\lambda_{2}-\lambda_{F}\right) n^{A}-\left(\lambda_{L}-\lambda_{2}\right) n^{B}}{\left(\lambda_{2}-\lambda_{1}\right)\left(\lambda_{L}-\lambda_{F}\right)}, \frac{\lambda_{2}\left(\lambda_{L}-\lambda_{F}\right) t-\left(\lambda_{1}-\lambda_{F}\right) n^{A}-\left(\lambda_{L}-\lambda_{1}\right) n^{B}}{\left(\lambda_{2}-\lambda_{1}\right)\left(\lambda_{L}-\lambda_{F}\right)}\right), & \text { if } X_{3}<t \leq X_{4}^{b}, \\ \left(\frac{n^{B}}{\lambda_{1}}, t\right), & \text { if } \min \left\{X_{2}^{b}, X_{4}^{b}\right\}<t \leq X_{6}, \quad \text { (VIII) } \\ \left(\frac{\lambda_{M 2} \lambda_{1} t-\lambda_{1} n^{A}-\left(\lambda_{M 1}-\lambda_{1}\right) n^{B}}{\lambda_{1}\left(\lambda_{M 2}-\lambda_{M 1}\right)}, t\right), & \text { if } X_{6}<t \leq X_{7}, \\ (t, t), & \text { if } X_{7}<t .\end{cases}
$$

The equilibrium payoffs in regions I, II, IV and V are same as those given in Eq. (23). The payoffs in region VIII are given as follows:

$\left(\boldsymbol{\Phi}^{A}, \boldsymbol{\Phi}^{B}\right)=\left(\frac{p_{2} \lambda_{1} \lambda_{M 2} t-\left(p_{2} \lambda_{M 2}-p_{1} \lambda_{1}\right) n^{B}}{\lambda_{1}}, p_{1} n^{B}\right)$

There are three differences between the characterizations in Theorem 2 and 1. First, region II is now defined as $X_{1}<t \leq$ min $\left\{X_{2}^{b}, X_{3}\right\}$. Second, region IV is changed to $X_{3}<t \leq X_{4}^{b}$. For a given season length $t$, regions II and IV are smaller in the space of $n^{A}$ and $n^{B}$. Third, regions III and V described in Theorem 1 disappear in favor of region VIII. Finally, one can show that in the parameter subspace defined by $n^{B} \geq \lambda_{1} t$ (which is a reasonable assumption, stating that neither firm can deplete its inventory if both firms keep the price high at $p_{1}$ ), the equilibria in Theorems 1 and 2 coincide.

Again, if we fix demand rates and season length, we can represent each region in Table 3 in a two-dimensional plot of $n^{A}$ and $n^{B}$. One example is provided in Fig. 2 for $\lambda_{1}=2 / 7, \lambda_{2}=4 / 7, \lambda_{F}=1 / 7, \lambda_{L}=5 / 7, \lambda_{M 1}=8 / 21, \lambda_{M 2}=1, p_{1}=10, p_{2}=6$ and $t=100$.

The characterization provided in Theorem 2 leads to the following corollaries.

Corollary 3. If $\chi_{3}<\lambda_{M 2} \leq \min \left\{\chi_{1}, \chi_{2}\right\}$, firm A marks down its price earlier than firm $B$ in equilibrium.

Corollary 4. If $\chi_{3}<\lambda_{M 2} \leq \min \left\{\chi_{1}, \chi_{2}\right\}$, in equilibrium, each firm follows one of four strategies:

(i) Mark the price down at the beginning of the season and sell as much inventory as possible at the low price.

(ii) Never mark the price down, and sell all inventory at the high price.

(iii) Mark the price down at a time such that all inventory is exhausted precisely at the end of the season.

(iv) Mark the price down right after the competitor runs out of stock.

Corollary 4 states that the property that is discussed in Corollary 2 for the case $\lambda_{M 2} \leq \min \left\{\chi_{1}, \chi_{2}, \chi_{3}\right\}$ now breaks. In equilibrium, a firm may switch its price after the season starts, but still end up with leftover inventory at the end of the season. In this case the firm that is interested in such a move is an overstocked firm and the smaller firm is clearly understocked. If the benefits of the rival firm running out of 


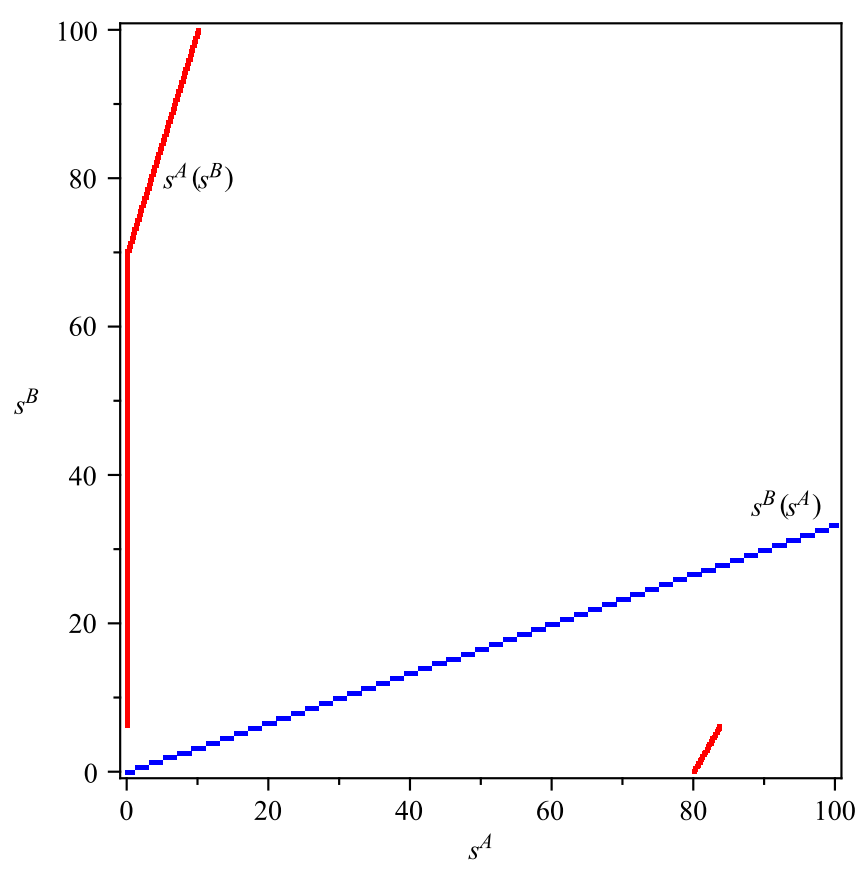

Fig. 3. Best response functions for Example 1.

stock is substantial, the overstocked firm may choose to postpone its markdown decision and wait until the rival firm runs out of stock, despite the fact that this does not eliminate the possibility of leftover inventory at the end.

Theorem 1 and 2 characterize equilibrium for each region under two cases, but do not guarantee the uniqueness of this equilibrium. We next provide a set of sufficient conditions for uniqueness which is valid for both cases.

Theorem 3. The equilibrium given in Theorems 1 and 2 are unique if

$\lambda_{2}\left(1+\min \left\{\frac{2 \lambda_{L}-\lambda_{1}-\lambda_{2}}{\lambda_{1}+\lambda_{2}-2 \lambda_{F}}, \frac{\lambda_{1}+\lambda_{2}-2 \lambda_{F}}{2 \lambda_{L}-\lambda_{1}-\lambda_{2}}\right\}\right)>\lambda_{M 2}$

$\left|\frac{\lambda_{1}\left(\lambda_{L}-\lambda_{F}\right)-\lambda_{M 1}\left(\lambda_{L}-\lambda_{1}\right)}{\lambda_{L}\left(\lambda_{M 2}-\lambda_{M 1}\right)}\right|<1$

We note that the bounds on $\lambda_{M 2}$ can be tighter or more relaxed than the bounds in $\chi_{1}, \chi_{2}$ and $\chi_{3}$ required for the equilibria in Theorem 1 and 2 .

\subsection{The case $\min \left\{\chi_{1}, \chi_{2}\right\}<\lambda_{M 2}$}

When $\lambda_{M 2}$ is allowed to be higher than either $\chi_{1}$ or $\chi_{2}$, we observe that a pure-strategy Nash equilibrium in general does not exist. We provide two examples, one for when $\chi_{2}>\lambda_{M 2}>\chi_{1}$ and one for when $\chi_{1}>\lambda_{M 2}>\chi_{2}$ (note again that $\lambda_{M 2}$ cannot be larger than both $\chi_{1}$ and $\left.\chi_{2}\right)$.

Example 1. Consider a game with $p_{1}=10, p_{2}=5.2, \lambda_{1}=2 / 7, \lambda_{2}=4 / 7, \lambda_{F}=1.7 / 7, \lambda_{L}=5 / 7, \lambda_{M 1}=0.5, \lambda_{M 2}=1.135, t=100, n^{A}=470 / 7$ and $n^{B}=400 / 7$. In this case, we have $\chi_{1}=0.989, \chi_{2}=2.769$ and $\chi_{3}=1.648$. We denote the best response function for firm $A$ as $s^{A}\left(s^{B}\right)$ and for firm $B$ as $s^{B}\left(s^{A}\right)$. These are plotted on opposite axes in Fig. 3. Since the functions do not intersect, there is no pure-strategy Nash equilibrium.

Example 2. Consider a game with $p_{1}=10, p_{2}=5.2, \lambda_{1}=2 / 7, \lambda_{2}=3 / 7, \lambda_{F}=0.5 / 7, \lambda_{L}=4.2 / 7, \lambda_{M 1}=0.3, \lambda_{M 2}=0.61, t=100, n^{A}=450 / 7$ and $n^{B}=250 / 7$. In this case, we have $\chi_{1}=1.157, \chi_{2}=0.530$ and $\chi_{3}=0.617$. The best response functions for this example are plotted in Fig. 4 . Again, since they do not intersect, there is no pure-strategy Nash equilibrium.

Remember that $\lambda_{M 2}>\chi_{1}$ or $\lambda_{M 2}>\chi_{2}$ makes monopolizing the total demand by forcing the rival firm to run out of inventory (even if this means finishing the season with leftover inventory) a more attractive option in comparison to executing the markdown when the rival firm is still in stock. This option is available to both firms regardless of how much inventory they have. These rather extreme benefits of rival firm's stock-out give rise to discontinuities in firms' response functions leading to non-existence of pure-strategy equilibrium. The destabilizing effect of a firm's increased demand due to a rival firm's stock-out can be considered to be similar to the effect of substitutability in Bertrand-Edgeworth-Chamberlin competition [4]. In both games, bounding the effect leads to the existence of the purestrategy equilibrium.

We note that by showing that the payoff functions defined by (8) are continuous in the switch times and by revoking a theorem by Glicksberg [15], one can prove the existence of a mixed-strategy Nash equilibrium for the markdown timing game. However the use of mixed-strategies in economic modeling and in particular, pricing decisions is controversial ([39], Section 2.4). Mixed-strategy equilibria 


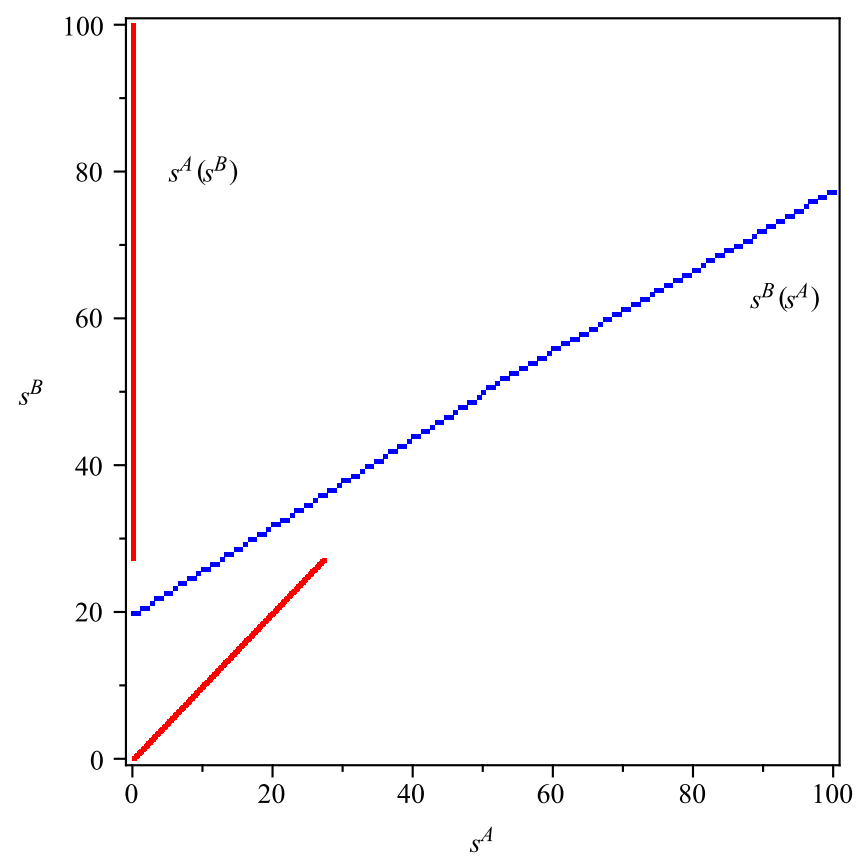

Fig. 4. Best response functions for Example 2.

are not stable (a player can, without any penalty, shift to any pure strategy which is assigned a positive probability in the equilibrium) and have the regret property (each player would like to wait and see what others played and respond in an optimal way; a player who plays earlier would regret its decision). Therefore, we focus our attention to pure-strategy equilibria in this paper.

We conclude this section by verifying that conditions (12)-(14) are satisfied if the demand rates originate from two demand functions introduced earlier in Section 3. First, we have the result for the linear demand model.

Proposition 2. There exists a unique pure-strategy Nash equilibrium for the markdown timing game for the linear demand model and the equilibrium is characterized by Theorem 1.

The following proposition characterizes the equilibrium for the attraction demand model.

Proposition 3. There exists a pure-strategy Nash equilibrium for the markdown timing game for the attraction demand model and it is characterized by Theorem 1. The equilibrium is unique if $a_{2}<3 a_{1}+\kappa$.

One can show that the condition in Proposition 3 is equivalent to $\lambda_{L}-\lambda_{F} \leq S / 2$, i.e., the difference between the demand rates of price leader and follower should be smaller than half of the total arrival rate (including the non-purchasers) for uniqueness.

\section{Comparative statics}

\subsection{Effect of inventory levels and selling season}

In this section, we study the effects of starting inventory levels and selling season on equilibrium behavior and payoffs. As shown in Theorem 1 and 2, there are many possible equilibria; we need to identify when the equilibrium switches from one region to another as the starting inventory levels and the length of the selling season change. First we study the case, $\lambda_{M 2} \leq \min \left\{\chi_{1}, \chi_{2}, \chi_{3}\right\}$. Analyzing all possible switches and changes inside each region of equilibria, we obtain the following comparative statics results.

Proposition 4. If $\lambda_{M 2} \leq \min \left\{\chi_{1}, \chi_{2}, \chi_{3}\right\}$, the following comparative statics results hold for the equilibrium switch times $\mathbf{s}^{A}$ and $\mathbf{s}^{B}$ and equilibrium payoffs $\boldsymbol{\Phi}^{\mathbf{A}}$ and $\boldsymbol{\Phi}^{\mathbf{B}}$ :

(a) $\mathbf{s}^{A}, \mathbf{s}^{B}, \boldsymbol{\Phi}^{\mathbf{A}}$ and $\boldsymbol{\Phi}^{\mathbf{B}}$ are increasing in $t$.

(b) $\mathbf{s}^{A}, \mathbf{s}^{B}$ and $\boldsymbol{\Phi}^{\mathbf{B}}$ are decreasing in $n^{A}$.

(c) $\mathbf{s}^{A}, \mathbf{s}^{B}$ and $\boldsymbol{\Phi}^{\mathbf{A}}$ are decreasing in $n^{B}$.

(d) $\boldsymbol{\Phi}^{\mathbf{A}}$ is decreasing in $n^{A}$ if $\frac{\lambda_{1}\left(\lambda_{L}-\lambda_{F}\right) t-\left(\lambda_{L}-\lambda_{1}\right) n^{B}}{\lambda_{1}-\lambda_{F}} \leq n^{A}<\frac{\lambda_{2}\left(\lambda_{L}-\lambda_{F}\right) t-\left(\lambda_{L}-\lambda_{2}\right) n^{B}}{\lambda_{2}-\lambda_{F}}$ and

$\left(p_{2} \lambda_{L}-p_{1} \lambda_{1}\right)\left(\lambda_{2}-\lambda_{1}\right)+\left(p_{2} \lambda_{2}-p_{1} \lambda_{1}\right)\left(\lambda_{1}-\lambda_{F}\right)<0$.

Otherwise, $\boldsymbol{\Phi}^{\mathbf{A}}$ is increasing in $n^{A}$.

(e) $\boldsymbol{\Phi}^{\mathbf{B}}$ is decreasing in $n^{B}$ if $\frac{\lambda_{1}\left(\lambda_{L}-\lambda_{F}\right) t-\left(\lambda_{1}-\lambda_{F}\right) n^{A}}{\lambda_{L}-\lambda_{1}} \leq n^{B}<\frac{\lambda_{2}\left(\lambda_{L}-\lambda_{F}\right) t-\left(\lambda_{2}-\lambda_{F}\right) n^{A}}{\lambda_{L}-\lambda_{2}}$ and

$\left(p_{2} \lambda_{2}-p_{1} \lambda_{F}\right)\left(\lambda_{2}-\lambda_{1}\right)+\left(p_{2} \lambda_{2}-p_{1} \lambda_{1}\right)\left(\lambda_{L}-\lambda_{2}\right)<0$.

Otherwise, $\boldsymbol{\Phi}^{\mathbf{B}}$ is increasing in $n^{B}$. 
While the results in parts (a)-(c) of Proposition 4 are intuitive, the exceptions in parts $(d)$ and $(e)$ are rather unexpected. Parts $(d)$ and (e) show that, in region IV, both firms may expect to get smaller payoffs as their own starting inventory gets larger. Before we explain this surprising comparative statics result, we note first that region IV is a plausible region for the firms. In fact, with a reasonable assumption that $\lambda_{2} t \geq n^{A} \geq n^{B} \geq \lambda_{1} t$, the parameter space consists of only region IV. In this region, both firms are endowed with intermediate levels of starting inventory (in comparison to season length) which they will exhaust precisely at the end of the season in equilibrium. Now first consider firm $A$, the larger firm. When the starting inventory of firm $A$ increases, it advances its markdown time. Since firm $B$ wants to exhaust its inventory as well, it responds by advancing its markdown time. As a result, both firms sell less of their inventory at full price. This leads to a reduction in payoff for firm $A$ if

$\frac{p_{2} \lambda_{L}-p_{1} \lambda_{1}}{\lambda_{1}-\lambda_{F}}<\frac{p_{1} \lambda_{1}-p_{2} \lambda_{2}}{\lambda_{2}-\lambda_{1}}$

The left-hand side of (29) is equal to the right-hand side of (10) and measures the increase in the revenue rate of the leader firm after its markdown per unit of decrease in the follower's demand rate. The right-hand side of (29) measures the difference between the revenue rates before and after two markdowns again per unit of increase in the demand rates.

Similarly, when the starting inventory of firm $B$ increases, it advances its markdown time followed by an advance in firm $A$ 's markdown time. As a result, again, both firms sell less of their inventory at full price. This results in a reduction in payoff for firm $B$ if

$\frac{p_{2} \lambda_{2}-p_{1} \lambda_{F}}{\lambda_{L}-\lambda_{2}}<\frac{p_{1} \lambda_{1}-p_{2} \lambda_{2}}{\lambda_{2}-\lambda_{1}}$

The left-hand side of (30) is equal to the right-hand side of (9) and measures the increase in the revenue rate of the follower firm after its markdown per unit of decrease in the leader's demand rate. Note that the two conditions (29) and (30) may hold simultaneously, i.e., both firms' payoffs may be non-monotone in their starting inventory levels in a given markdown timing game. When $p_{2} \lambda_{2}>p_{1} \lambda_{1}$, i.e., if the revenue rate is increasing even when the price change is matched by the competitor, the right-hand sides of (29) and (30) become negative and this interesting behavior disappears.

These negative results on the monotonicity of firms' payoffs show that even when firms' demands are elastic to unilateral markdowns $\left(p_{2} \lambda_{L}-p_{1} \lambda_{1} \geq 0\right.$ and $\left.p_{2} \lambda_{2}-p_{1} \lambda_{F} \geq 0\right)$, if they are inelastic to an industry-wide markdown $\left(p_{2} \lambda_{2}<p_{1} \lambda_{1}\right)$, engaging in markdown competition may lead to obtaining less revenue for more inventory (See [6] for a few examples of such industries). In these cases, firms may even consider salvaging some of their inventory prior to markdown competition. This would obviously decrease their sales in quantity, but their total revenues would go up. This comparative statics result also means that, given that firms cannot internalize their markdown prices and face deterministic demands, they may not prefer to have more inventory even if it is free. A similar comparative statics result is obtained in Osborne and Pitchik [23] for the Bertrand-Edgeworth competition where the profit for the smaller firm may decrease as its capacity increases. Our results are more striking since monotonicity may break for both firms simultaneously in our case and we consider the total revenues. In practice, these results show that strategic interactions between competing firms may have a detrimental effect on profitability of both firms especially when markdowns provide only a temporary revenue jump until the rival firm matches the price. In this case, having more inventory in either firm may actually reduce the total revenue of both firms. The hit on revenue per unit or profit per unit will be even more substantial.

For the case $\chi_{3}<\lambda_{M 2} \leq \min \left\{\chi_{1}, \chi_{2}\right\}$, we obtain the following comparative statics results.

Proposition 5. If $\chi_{3}<\lambda_{M 2} \leq \min \left\{\chi_{1}, \chi_{2}\right\}$, the following comparative statics results hold for the equilibrium switch times $\mathbf{s}^{A}$ and $\mathbf{s}^{B}$ and equilibrium payoffs $\boldsymbol{\Phi}^{\mathbf{A}}$ and $\boldsymbol{\Phi}^{\mathbf{B}}$.

(a) $\mathbf{s}^{A}, \mathbf{s}^{B}, \boldsymbol{\Phi}^{\mathbf{A}}$ and $\mathbf{\Phi}^{\mathbf{B}}$ are increasing in $t$.

(b) $\mathbf{s}^{A}, \mathbf{s}^{B}$ and $\mathbf{\Phi}^{\mathbf{B}}$ are decreasing in $n^{A}$.

(c) $\mathbf{s}^{B}$ and $\boldsymbol{\Phi}^{A}$ are decreasing in $n^{B}$.

(d) $\boldsymbol{\Phi}^{\mathbf{A}}$ is decreasing in $n^{A}$ if $\frac{\lambda_{1}\left(\lambda_{L}-\lambda_{F}\right) t-\left[\left(\lambda_{L}-\lambda_{F}\right)-\vartheta \lambda_{1}\right] n^{B}}{\vartheta \lambda_{1}}<n^{A} \leq \frac{\lambda_{2}\left(\lambda_{L}-\lambda_{F}\right) t-\left(\lambda_{L}-\lambda_{2}\right) n^{B}}{\lambda_{2}-\lambda_{F}}$, and

$\left(p_{2} \lambda_{L}-p_{1} \lambda_{1}\right)\left(\lambda_{2}-\lambda_{1}\right)+\left(p_{2} \lambda_{2}-p_{1} \lambda_{1}\right)\left(\lambda_{1}-\lambda_{F}\right)<0$

Otherwise, $\boldsymbol{\Phi}^{\mathbf{A}}$ is increasing in $n^{A}$.

(e) $\boldsymbol{\Phi}^{\mathbf{B}}$ is increasing in $n^{B}$, with the following exceptions:

(i) It is decreasing in $n^{B}$ when $\frac{\lambda_{1}\left(\lambda_{L}-\lambda_{F}\right) t-\vartheta \lambda_{1} n^{A}}{\left(\lambda_{L}-\lambda_{F}\right)-\vartheta \lambda_{1}}<n^{B} \leq \frac{\lambda_{2}\left(\lambda_{L}-\lambda_{F}\right) t-\left(\lambda_{2}-\lambda_{F}\right) n^{A}}{\lambda_{L}-\lambda_{2}}$, and

$\left(p_{2} \lambda_{2}-p_{1} \lambda_{F}\right)\left(\lambda_{2}-\lambda_{1}\right)+\left(p_{2} \lambda_{2}-p_{1} \lambda_{1}\right)\left(\lambda_{L}-\lambda_{2}\right)<0$.

(ii) It jumps down at $n^{B}=\varphi t$ and $n^{B}=\frac{\lambda_{1}\left(\lambda_{L}-\lambda_{F}\right) t-9 \lambda_{1} n^{A}}{\left(\lambda_{L}-\lambda_{F}\right)-\vartheta \lambda_{1}}$.

(f) $\mathbf{s}^{A}$ is increasing in $n^{B}$ if $\min \left\{\varphi t, \frac{\lambda_{1}\left(\lambda_{L}-\lambda_{F}\right) t-9 \lambda_{1} n^{A}}{\left(\lambda_{L}-\lambda_{F}\right)-\vartheta \lambda_{1}}\right\}<n^{B} \leq \frac{\lambda_{1} \lambda_{M 2} t-\lambda_{1} n^{A}}{\lambda_{M 2}-\lambda_{1}}$. Otherwise, $\mathbf{s}^{A}$ is decreasing in $n^{B}$.

Parts (a)-(c) of Proposition 5 are again intuitive and expected. The exception in part (d) and exception (i) in part (e) state the behavior in region IV and are also observed and discussed for the case $\lambda_{M 2} \leq\left\{\chi_{1}, \chi_{2}, \chi_{3}\right\}$. Exception (ii) in part (e) is due to the switches from regions II or IV to region VIII, and states a third case where larger inventories may in fact lead to lower payoffs. Note that in region VIII, the starting inventory of the smaller firm is excessively low. The non-monotonicity result here shows that the smaller firm prefers buffering of the larger firm to engaging in a markdown competition and would therefore be better off if its inventory is smaller. Part (f) is due to the equilibrium strategy of firm $A$ in region VIII. Since $A$ waits until firm $B$ runs out of stock in region VIII, higher starting inventory for firm $B$ leads to a later switch time for firm $A$. This shows that more industry-level inventory may not always lead to earlier markdowns in the industry. This also leads to larger equilibrium prices which is in contrast to the behavior in classical price oligopolies. 


\subsection{Effect of product substitutability and inventory imbalance}

We now turn our attention to the impact of product substitutability and inventory imbalance on equilibrium switch times. We focus on equilibrium in region IV as characterized in Theorem 1 and 2 because the switch times for both firms are inside the season only in this region of parameter space. We should also note that under a rather reasonable assumption that $\lambda_{1} t \leq n^{B} \leq n^{A} \leq \lambda_{2} t$, we only have region IV, and the switch times

$\left(\mathbf{s}^{A}, \mathbf{s}^{B}\right)=\left(\frac{\lambda_{2}\left(\lambda_{L}-\lambda_{F}\right) t-\left(\lambda_{2}-\lambda_{F}\right) n^{A}-\left(\lambda_{L}-\lambda_{2}\right) n^{B}}{\left(\lambda_{2}-\lambda_{1}\right)\left(\lambda_{L}-\lambda_{F}\right)}, \frac{\lambda_{2}\left(\lambda_{L}-\lambda_{F}\right) t-\left(\lambda_{1}-\lambda_{F}\right) n^{A}-\left(\lambda_{L}-\lambda_{1}\right) n^{B}}{\left(\lambda_{2}-\lambda_{1}\right)\left(\lambda_{L}-\lambda_{F}\right)}\right)$

is the only equilibrium of the game.

From (31), the length of the period during which the firms charge different prices equals

$\mathbf{s}^{B}-\mathbf{s}^{A}=\frac{n^{A}-n^{B}}{\lambda_{L}-\lambda_{F}}$.

Note that the formula describes two forces in action. While the difference in starting inventory levels $\left(n^{A}-n^{B}\right)$ forces the firms to set their markdown times far apart from each other, product substitutability $\left(\lambda_{L}-\lambda_{F}\right)$ forces the firms to reduce the difference between markdown times. As products become less differentiated $\left(\lambda_{L}-\lambda_{F}\right.$ increases), firms tend to follow each other's decisions, and switch at closer times to each other. These two forces are simplifications of two general phenomena. While the difference in firm sizes leads firms to follow different strategies, product substitutability and competing in the same market limits them in doing so.

For the linear demand model, Eq. (32) is equal to

$\mathbf{s}^{B}-\mathbf{s}^{A}=\frac{\epsilon\left(n^{A}-n^{B}\right)}{p_{1}-p_{2}}$

which shows that the length of the period during which firms charge different prices is increasing in the degree of product differentiation

$(\epsilon)$ and decreasing in the difference of initial and markdown prices.

For the attraction demand model, Eq. (32) is equal to

$\mathbf{s}^{B}-\mathbf{s}^{A}=\frac{\left(a_{1}+a_{2}+\kappa\right)\left(n^{A}-n^{B}\right)}{\left(a_{2}-a_{1}\right) S}$,

which shows that the length of the period during which firms charge different prices is increasing in the no purchase probability ( $\kappa$ ) and decreasing in the difference of initial and markdown prices.

\section{Closed-loop equilibrium}

In this section, we relax the assumption that firms pre-commit themselves to markdown times at the beginning of the sales horizon and consider closed-loop strategies. For this analysis, we study a smaller subset of the parameter space and assume that $\lambda_{2} t \geq n^{A} \geq n^{B} \geq \lambda_{1} t$ (only region IV). Under this reasonable assumption, each firm can finish their inventory if both firms charge the low price throughout the season. In contrast, when both firms charge the high firm, neither firm can finish its inventory. We formulate the game as a simple timing game where each firm's only choice is when to mark the price down ([9], Section 4.5). When a firm marks the price down, it has no effect on the future of the game. If a firm did not change the price at any time before $\varsigma$, its action set at time $\varsigma$ is $A^{i}(\varsigma)=\{$ "mark down", "do not mark down" $\}$. If firm $i$ has marked the price down at some time before $\varsigma$, then its action set is the null action.

The natural solution concept for simple timing games is subgame-perfect equilibrium. In this case, once one of the firms (leader) has marked the price down, the other firm (follower) faces a maximization problem (i.e., continuation problem) which can be solved relatively easily. Therefore we can express both firms' payoffs as functions of the time

$s=\min \left\{\varsigma \mid a^{i}(\varsigma)=\right.$ "mark down", for at least one $\left.i\right\}$,

which is the time at which the first firm marks the price down (These best response functions can be characterized by solving the continuation problem). Then, we can represent firm $A^{\prime}$ s payoff function as a function of the first switch time as $L^{A}(s)=\Phi^{A}\left(s, r^{B}(s)\right)$ if it is the leader and $F^{A}(s)=\Phi^{A}\left(r^{A}(s), s\right)$ if it is the follower. Similarly, firm $B^{\prime}$ s payoff function is $L^{B}(s)=\Phi^{B}\left(r^{A}(s), s\right)$ if it is the leader and $F^{B}(s)=\Phi^{B}$ $\left(s, r^{B}(s)\right)$ if it is the follower.

The next theorem characterizes these payoff functions ([ $\left[\frac{x}{y}\right]^{\diamond}$ denotes $\frac{x}{y}$ if $x \geq 0$ and $y>0$ and 0 , otherwise).

Theorem 4. The payoffs for firms $A$ and $B$ are given as follows:

$F^{B}(s)= \begin{cases}\zeta_{1}\left(t, n^{B}, n^{A}\right)-\left(p_{2} \lambda_{2}-p_{1} \lambda_{1}\right) s, & \text { if } s \geq \bar{s}_{1}^{A}, \\ \zeta_{2}^{a}\left(t, n^{B}, n^{A}\right)+\frac{\lambda_{2}\left(p_{1}-p_{2}\right)\left(\lambda_{1}-\lambda_{F}\right)}{\lambda_{2}-\lambda_{F}} s, & \text { if } \bar{s}_{1}^{A}>s \geq \bar{s}_{2}^{A}, \\ \zeta_{3}\left(t, n^{B}, n^{A}\right)+\frac{\left(p_{1}-p_{2}\right)\left[\left(\lambda_{L}-\lambda_{F}\right) \lambda_{1} \lambda_{2}-\lambda_{M 2}\left[\lambda_{1}\left(\lambda_{L}-\lambda_{2}\right)+\lambda_{F}\left(\lambda_{2}-\lambda_{1}\right)\right]\right]}{\lambda_{2}\left(\lambda_{L}-\lambda_{F}\right)-\lambda_{M 2}\left(\lambda_{L}-\lambda_{2}\right)} s, & \text { if } \bar{s}_{2}^{A}>s \geq \bar{s}_{3}^{A}, \\ \zeta_{4}\left(t, n^{B}, n^{A}\right)+\frac{\left(p_{1}-p_{2}\right) \lambda_{M 2}\left[\lambda_{1}\left(\lambda_{L}-\lambda_{F}\right)-\lambda_{M 1}\left(\lambda_{L}-\lambda_{1}\right)\right]}{\lambda_{L}\left(\lambda_{M 2}-\lambda_{M 1}\right)} s & \text { if } \bar{s}_{3}^{A}>s \geq \bar{s}_{4}^{A}, \\ \zeta_{5}\left(t, n^{B}, n^{A}\right), & \text { if } s<\bar{s}_{4}^{A},\end{cases}$ 


$$
\begin{gathered}
L^{A}(s)=\left\{\begin{array}{lr}
p_{2} \lambda_{2} t-\left(p_{2} \lambda_{2}-p_{1} \lambda_{1}\right) s, \\
\frac{p_{2} \lambda_{2}\left(\lambda_{L}-\lambda_{F}\right) t-p_{2}\left(\lambda_{L}-\lambda_{2}\right) n^{B}}{\lambda_{2}-\lambda_{F}}-\frac{\left(p_{2} \lambda_{2}-p_{1} \lambda_{1}\right)\left(\lambda_{1}-\lambda_{F}\right)+\left(p_{2} \lambda_{L}-p_{1} \lambda_{1}\right)\left(\lambda_{2}-\lambda_{1}\right)}{\lambda_{2}-\lambda_{F}} s, & \text { if } s \geq \bar{s}_{1}^{A}, \\
p_{2} n^{A}+\left(p_{1}-p_{2}\right) \lambda_{1} s & \text { if } s<\bar{s}_{2}^{A},
\end{array}\right. \\
F^{A}(s)= \begin{cases}\zeta_{1}\left(t, n^{A}, n^{B}\right)-\left(p_{2} \lambda_{2}-p_{1} \lambda_{1}\right) s, & \text { if } s \geq \bar{s}_{1}^{B}, \\
\zeta_{2}^{b}\left(t, n^{A}, n^{B}\right)+\frac{\lambda_{1} \lambda_{2}\left(p_{1}-p_{2}\right)-p_{2} \lambda_{M 2}\left(\lambda_{2}-\lambda_{1}\right)}{\lambda_{2}} s, & \text { if } \bar{s}_{1}^{B}>s \geq \bar{s}_{2}^{B}, \\
\zeta_{3}\left(t, n^{A}, n^{B}\right)+\frac{\left(p_{1}-p_{2}\right)\left[\left(\lambda_{L}-\lambda_{F}\right) \lambda_{1} \lambda_{2}-\lambda_{M 2}\left[\lambda_{1}\left(\lambda_{L}-\lambda_{2}\right)+\lambda_{F}\left(\lambda_{2}-\lambda_{1}\right)\right]\right]}{\lambda_{2}\left(\lambda_{L}-\lambda_{F}\right)-\lambda_{M 2}\left(\lambda_{L}-\lambda_{2}\right)} s, & \text { if } \bar{s}_{2}^{B}>s \geq \bar{s}_{3}^{B}, \\
\zeta_{4}\left(t, n^{A}, n^{B}\right)+\frac{\left(p_{1}-p_{2}\right) \lambda_{M 2}\left[\lambda_{1}\left(\lambda_{L}-\lambda_{F}\right)-\lambda_{M 1}\left(\lambda_{L}-\lambda_{1}\right)\right]}{\lambda_{L}\left(\lambda_{M 2}-\lambda_{M 1}\right)} s, & \text { if } \bar{s}_{3}^{B}>s \geq \bar{s}_{4}^{B}, \\
\zeta_{5}\left(t, n^{A}, n^{B}\right), & \text { if } s \leq \bar{s}_{4}^{B},\end{cases} \\
L^{B}(s)= \begin{cases}p_{2} \lambda_{2} t-\left(p_{2} \lambda_{2}-p_{1} \lambda_{1}\right) s & \text { if } s \geq \bar{s}_{1}^{A}, \\
p_{2} n^{B}+\left(p_{1}-p_{2}\right) \lambda_{1} s & \text { if } s<\bar{s}_{1}^{A},\end{cases}
\end{gathered}
$$

where

$\zeta_{1}\left(\tau, \nu_{F}, \nu_{L}\right)=p_{2} \lambda_{2} \tau$,

$\zeta_{2}^{a}\left(\tau, \nu_{F}, \nu_{L}\right)=\frac{\left(p_{1}-p_{2}\right) \lambda_{2} \lambda_{F} \tau+\left(p_{2} \lambda_{2}-p_{1} \lambda_{F}\right) \nu_{F}}{\lambda_{2}-\lambda_{F}}$,

$\zeta_{2}^{b}\left(\tau, \nu_{F}, \nu_{L}\right)=p_{2} \lambda_{M 2} \tau-p_{2} \frac{\left(\lambda_{M 2}-\lambda_{2}\right)}{\lambda_{2}} \nu_{L}$,

$\zeta_{3}\left(\tau, \nu_{F}, \nu_{L}\right)=\frac{\left(p_{1}-p_{2}\right) \lambda_{2} \lambda_{F} \lambda_{M 2} \tau-\left[p_{1} \lambda_{F} \lambda_{2}+p_{2}\left(\lambda_{L}\left(\lambda_{M 2}-\lambda_{2}\right)-\lambda_{2} \lambda_{M 2}\right)\right] \nu_{F}}{\lambda_{2}\left(\lambda_{L}-\lambda_{F}\right)-\lambda_{M 2}\left(\lambda_{L}-\lambda_{2}\right)}-\frac{\left(p_{1}-p_{2}\right) \lambda_{F}\left(\lambda_{M 2}-\lambda_{2}\right) \nu_{L}}{\lambda_{2}\left(\lambda_{L}-\lambda_{F}\right)-\lambda_{M 2}\left(\lambda_{L}-\lambda_{2}\right)}$,

$\zeta_{4}\left(\tau, \nu_{F}, \nu_{L}\right)=\frac{\left(p_{1}-p_{2}\right) \lambda_{M 1} \lambda_{M 2} \lambda_{L} \tau+\left(p_{2} \lambda_{M 2}-p_{1} \lambda_{M 1}\right) \lambda_{L} \nu_{F}-\left(p_{1}-p_{2}\right) \lambda_{M 2}\left(\lambda_{M 1}-\lambda_{F}\right) \nu_{L}}{\lambda_{L}\left(\lambda_{M 2}-\lambda_{M 1}\right)}$,

$\zeta_{5}\left(\tau, \nu_{F}, \nu_{L}\right)=p_{1} \nu_{F}$,

and

$\bar{s}_{1}^{A}=\frac{\lambda_{2} t-n^{B}}{\lambda_{2}-\lambda_{1}}$,

$\bar{s}_{2}^{A}=\frac{\lambda_{2}\left(\lambda_{L}-\lambda_{F}\right) t-\left(\lambda_{L}-\lambda_{2}\right) n^{B}-\left(\lambda_{2}-\lambda_{F}\right) n^{A}}{\left(\lambda_{L}-\lambda_{F}\right)\left(\lambda_{2}-\lambda_{1}\right)}$,

$\bar{s}_{3}^{A}=\left[\frac{\lambda_{L} \lambda_{M 2} t-\lambda_{L} n^{B}-\left(\lambda_{M 2}-\lambda_{F}\right) n^{A}}{\left(\lambda_{M 2}-\lambda_{1}\right) \lambda_{L}-\left(\lambda_{M 2}-\lambda_{F}\right) \lambda_{1}}\right]^{\diamond}$

$\bar{s}_{4}^{A}=\left[\frac{\lambda_{L} \lambda_{M 1} t-\lambda_{L} n^{B}-\left(\lambda_{M 1}-\lambda_{F}\right) n^{A}}{\left(\lambda_{M 1}-\lambda_{1}\right) \lambda_{L}-\left(\lambda_{M 1}-\lambda_{F}\right) \lambda_{1}}\right]^{\diamond}$,

$\bar{s}_{1}^{B}=\frac{\lambda_{2} t-n^{B}}{\lambda_{2}-\lambda_{1}}$,

$\bar{s}_{2}^{B}=\frac{\lambda_{2} \lambda_{M 2} t-\lambda_{2} n^{A}-\left(\lambda_{M 2}-\lambda_{2}\right) n^{B}}{\lambda_{M 2}\left(\lambda_{2}-\lambda_{1}\right)}$,

$\bar{s}_{3}^{B}=\left[\frac{\lambda_{L} \lambda_{M 2} t-\lambda_{L} n^{A}-\left(\lambda_{M 2}-\lambda_{F}\right) n^{B}}{\left(\lambda_{M 2}-\lambda_{1}\right) \lambda_{L}-\left(\lambda_{M 2}-\lambda_{F}\right) \lambda_{1}}\right]^{\diamond}$,

$\bar{s}_{4}^{B}=\left[\frac{\lambda_{L} \lambda_{M 1} t-\lambda_{L} n^{A}-\left(\lambda_{M 1}-\lambda_{F}\right) n^{B}}{\left(\lambda_{M 1}-\lambda_{1}\right) \lambda_{L}-\left(\lambda_{M 1}-\lambda_{F}\right) \lambda_{1}}\right]^{\diamond}$

An example for the payoff functions is given in Fig. 5.

We now show that the open-loop equilibrium found in Section 4 is also the closed-loop equilibrium. In order words, there is no motivation for the firms to preempt each other in the timing game. First, notice that all payoff functions given by Eqs. (33)-(36) are continuous functions of the first switch time. In addition, the leader payoff function for firm $A$, given in Eq. (34) is single peaked at $\bar{s}_{2}^{A}$. The leader payoff function for firm $B$, given in Eq. (36) is also single peaked, but at $\bar{s}_{1}^{B}$. Notice that $\bar{s}_{1}^{B}=\bar{s}_{1}^{A}$ and $\bar{s}_{2}^{A} \leq \bar{s}_{1}^{A}$. Therefore, firm $A$ would want to lead and change the price to $p_{2}$ at time $\bar{s}_{2}^{A}$. However, one needs to ensure that firm $B$ would not preempt firm $A$. This can be shown by showing that the function $F^{B}$ is strictly increasing and is larger than the function $L^{B}$ at $\bar{s}_{2}^{A}$. One can see that $F^{B}$ is increasing between $\bar{s}_{3}^{A}$ and $\bar{s}_{1}^{A}$ from Eq. (33). One can also show that when $\varsigma \in\left[\bar{s}_{2}^{A}, \bar{s}_{1}^{A}\right)$

$F^{B}(\varsigma)-L^{B}(s)=\frac{\left(p_{1}-p_{2}\right) \lambda_{F}\left(\lambda_{2} t-n^{B}\right)}{\lambda_{2}-\lambda_{F}}-\frac{\left(p_{1}-p_{2}\right)\left(\lambda_{2}-\lambda_{1}\right) \lambda_{F}}{\lambda_{2}-\lambda_{F}} s$, 


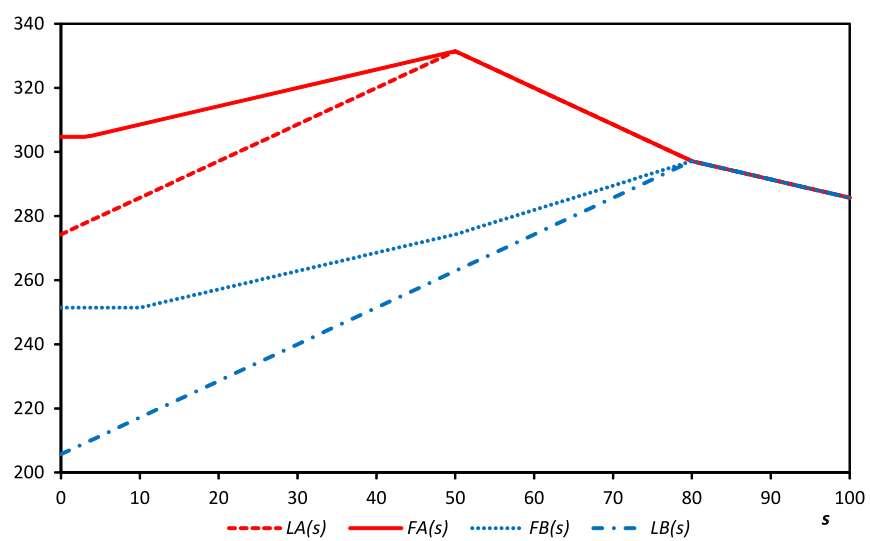

Fig. 5. Leader and Follower payoff functions: $\lambda_{F}=1 / 7, \lambda_{1}=2 / 7, \lambda_{2}=4 / 7, \lambda_{L}=5 / 7, \lambda_{M 1}=8 / 21, \lambda_{M 2}=16 / 21, p_{1}=10, p_{2}=6, n^{A}=320 / 7, n^{B}=240 / 7$.

and

$F^{B}\left(\bar{s}_{2}^{A}\right)-L^{B}\left(\bar{s}_{2}^{A}\right)=\frac{\lambda_{F}\left(p_{1}-p_{2}\right)\left(n^{A}-n^{B}\right)}{\lambda_{L}-\lambda_{F}}$

which is strictly positive if $n^{A}>n^{B}$. Therefore firm $B$ has no motivation to preempt. Therefore firm $A$ switches at $\bar{s}_{2}^{A}=\mathbf{s}^{A}$. The solution of firm $B$ 's switch time is found by solving the continuation problem. The result is given in the following theorem.

Theorem 5. When $n^{A} \geq n^{B}$, the equilibrium given by the switch times

$\mathbf{s}^{A}=\frac{\lambda_{2}\left(\lambda_{L}-\lambda_{F}\right) t-\left(\lambda_{L}-\lambda_{2}\right) n^{B}-\left(\lambda_{2}-\lambda_{F}\right) n^{A}}{\left(\lambda_{2}-\lambda_{1}\right)\left(\lambda_{L}-\lambda_{F}\right)}$

$\mathbf{s}^{B}=\frac{\lambda_{2}\left(\lambda_{L}-\lambda_{F}\right) t-\left(\lambda_{L}-\lambda_{1}\right) n^{B}-\left(\lambda_{1}-\lambda_{F}\right) n^{A}}{\left(\lambda_{2}-\lambda_{1}\right)\left(\lambda_{L}-\lambda_{F}\right)}$

is also a closed-loop equilibrium.

The result may be expected since the demands are deterministic. In addition switching price first does not lead to any permanent firstmover advantage. If a firm preempts the other firm and switch the price early, the other firm can simply match the price. We conjecture that similar results are valid for other regions of the parameter space.

\section{Conclusion}

In this paper, we study the effects of competition on markdown timing decisions of two firms with fixed inventories. The model we use is admittedly simple and was chosen to understand strategic interactions between competing firms and obtain important insights, rather predicting the outcome of a specific game played in a particular setting. We show that a pure-strategy Nash equilibrium exists under certain conditions and its specific characterization depends on the inventory levels, the length of the selling season and the demand rates. In general, in equilibrium, if a firm marks its price down after the season starts, it ensures that it runs out of inventory precisely when the season ends. An exception is when the smaller firm is substantially small and the larger firm's demand increases significantly when the smaller firm's inventory runs out. In this case, the larger firm has an incentive to hold its markdown until its competitor runs out of stock despite the fact that this would lead to having unsold inventory at the end of season.

We show that usual monotonicity results may fail to hold in a competitive environment. In particular, both firms' revenue may be nonmonotone in their own starting inventory, i.e., more inventory may lead to less revenue. We also show that the duration of price dispersion can be explained by two factors: inventory imbalance and product substitutability. The time period over which firms charge different prices increases linearly with inventory imbalance and decreases reciprocally with product substitutability. We finally show that the conditions for the existence and uniqueness of an equilibrium are easily satisfied for the case of two commonly used price-response functions.

One can consider extending the model in this paper in many different directions. Asymmetry in firms' prices and demand rates complicate the analysis, but do not appear to introduce any insight that is qualitatively different from what we obtain here (asymmetry in demand rates can be easily handled for the linear demand function in the current model). Another direction would be to study closed-loop strategies in all regions of the parameter space. Our results with a reasonable subset of the parameter space show that open-loop equilibrium survive as an equilibrium in this dynamic game. This is due to the fact that the demands are deterministic and the fact that being a price leader does not introduce any permanent first-mover advantage. Therefore, we conjecture this will be true for other regions as well. Studying the markup problem (which is more in line with capacity allocation in many revenue management industries) under a similar setting would be another future direction. We believe that analysis and the insights from that research would be similar to what we provide here.

It may be worthwhile to introduce the depth of the markdown as a second strategic variable. It would be also interesting to see the impact of incomplete information, particularly on starting inventory levels. These two extensions would bring the model closer to reality and may provide additional insights. One final important extension would be to model uncertainty in demand. However, given that the 
optimal policy can be computed only algorithmically [7] even for the monopolistic case, it may be difficult to obtain insights regarding the strategic behavior of firms in these settings using a stochastic model.

\section{Appendix A. Supplementary data}

Supplementary data associated with this article can be found in the online version at http://dx.doi.org/10.1016/j.omega.2015.10.016.

\section{References}

[1] Automotive News. Ford cuts prices on restyled 2007 Expedition. July 31; vol. 80, 2006. p. 8.

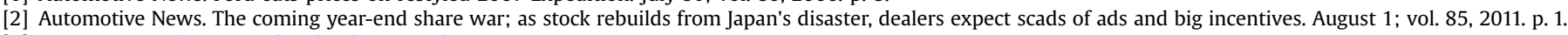

[3] Barrett M. Tracing apparel technology trends. Appar Mag 2007;48:2-16.

[4] Benassy J-P. Market size and substitutability in imperfect competition: a Bertrand-Edgeworth-Chamberlin model. Rev Econ Stud 1989;56:217-234.

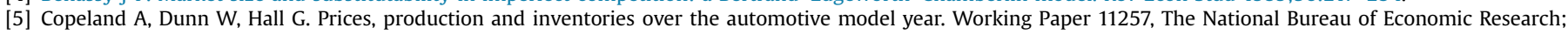
2005.

[6] Dolan Robert. Models of competition: a review of theory and empirical evidence. Rev Mark 1981;6:224-234.

[7] Feng Y, Gallego G. Optimal starting times for end-of-season sales and optimal stopping times for promotional fares. Manag Sci 1995;41:1371-1391.

[8] Fisher M, Raman A. Reducing the cost of demand uncertainty through accurate response to early sales. Oper Res 1996;44:87-99.

[9] Fudenberg D, Tirole J. Game theory. Cambridge, Massachusetts. The MIT Press; 1991.

[10] Gallego G, Hu M. Dynamic pricing of perishable assets under competition. Technical Report, Columbia University; 2013.

[11] Gallego G, van Ryzin G. Optimal dynamic pricing of inventories with stochastic demand over finite horizons. Manag Sci 1994;40:999-1020.

[12] Gallego G, van Ryzin G. A multiproduct dynamic pricing problem and its applications to network yield management. Oper Res 1997;45:24-41.

[13] Ghemawat P, McGahan AM. Order backlogs and strategic pricing: the case of the U.S. large turbine generator industry. Strateg Manag J 1998;19:255-268.

[14] Ghemawat P, Nalebuff B. Exit. The RAND. J Econ 1985;16:184-194.

[15] Glicksberg IL. A further generalization of the Kakutani fixed point theorem, with application to Nash equilibrium points. Proc Am Math Soc 1952;3:170-174.

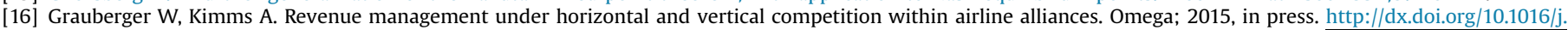
omega.2015.06.010.

[17] Lazear EP. Retail pricing and clearance sales. Am Econ Rev 1986;76:14-32.

[18] Levin Y, McGill J, Nediak M. Dynamic pricing in the presence of strategic consumers and oligopolistic competition. Manag Sci 2009;55:32-46.

[19] Lin KY, Sibdari SY. Dynamic price competition with discrete customer choices. Eur J Oper Res 2009;197:969-980.

[20] Los Angeles Times. J.C. Penney to adapt simple pricing strategy. January 26; 2012. p. B5.

[21] Martinez-de Albeniz V, Talluri K. Dynamic price competition with fixed capacities. Manag Sci 2011;57:1078-1093.

[22] Netessine S, Shumsky RA. Revenue management games: horizontal and vertical competition. Manag Sci 2005;51:813-831.

[23] Osborne MJ, Pitchik C. Price competition in a capacity-constrained duopoly. J Econ Theory 1986;38:238-260.

[24] Pashigian BP. Demand uncertainty and sales: a study of fashion and markdown pricing. Am Econ Rev 1988;78:936-953.

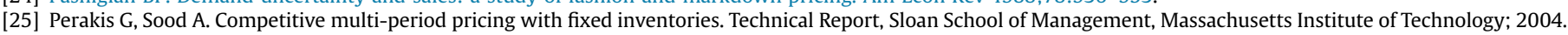

[26] Perakis G, Sood A. Competitive multi-period pricing for perishable products: a robust optimization approach. Math Progr 2006;107:295-335.

[27] Reinganum JF. On the diffusion of new technology: a game theoretic approach. Rev Econ Stud 1981;48:395-405.

[28] Sen A. The US fashion industry: a supply chain review. Int J Prod Econ 2008;114:571-593.

[29] Sen A. A comparison of fixed and dynamic pricing policies in revenue management. Omega 2013;41:586-597.

[30] Shapley L, Shubik M. Price strategy oligopoly with product variation. Kyklos 1969;22:30-44.

[31] Stores. Pricing transparency: clarity requires science, art and psychology. February; 2012.

[32] Talluri K. On equilibria in duopoloes with finite strategy spaces. Technical Report, Universitat Pompeu Fabra; 2003.

[33] Talluri K, van Ryzin G. The theory and practice of revenue management. International series in operations research \& management science, vol. 68. Springer; 2005.

[34] The Wall Street Journal. Retailers feel squeezed on the margin. June 8; 2011. Online edition.

[35] The Wall Street Journal. J.C. Penney gets rid of hundreds of sales. January 25; 2012. Online edition.

[36] The Washington Post. In electronics, retailers start dealing early. November 15; 2010. p. A7.

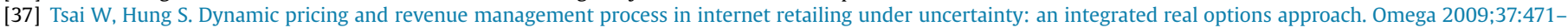
481.

[38] USA Today. Stores plan to keep hot toys in stock; inventory caution seems lighter than last holiday. October 6; 2010. Money, 1B.

[39] Vives X. Oligopoly pricing: old ideas and new tools. Cambridge, Massachusetts: MIT Press; 1999.

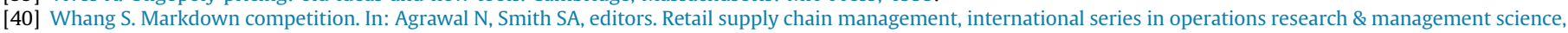
vol. 122. Springer; 2009. p. 293-307.

[41] Xu X, Hopp WJ. A monopolistic and oligopolistic stochastic flow revenue management model. Oper Res 2006;54:1098-1109.

[42] Yao D, Liu JJ. Competitive pricing of mixed retail and e-tail distribution channels. Omega 2005;33:235-247. 